\title{
INPP5D Expression is Associated with Risk for Alzheimer's Disease and Induced by Plaque- Associated Microglia
}

\section{Andy P Tsai}

Stark Neurosciences Research Institute

\section{Peter Bor-Chian Lin}

Stark Neurosciences Research Institute

Chuanpeng Dong

Indiana University School of Medicine

Miguel Moutinho

Stark Neurosciences Research Institute

\section{Brad Casali}

Case Western Reserve University

\section{Yunlong Liu}

Indiana University School of Medicine

\section{Bruce T Lamb}

Stark Neurosciences Research Institute

\section{Gary E Landreth}

Stark Neurosciences Research Institute

\section{Adrian L Oblak}

Stark Neurosciences Research Institute

Kwangsik Nho ( $\nabla$ knho@iupui.edu )

Indiana University School of Medicine

\section{Research article}

Keywords: Alzheimer's disease (AD), Microglia, INPP5D, AD risk, Plaque

Posted Date: September 8th, 2020

DOI: https://doi.org/10.21203/rs.3.rs-69967/v1

License: (a) (i) This work is licensed under a Creative Commons Attribution 4.0 International License.

Read Full License 
Version of Record: A version of this preprint was published at Neurobiology of Disease on June 1st, 2021. See the published version at https://doi.org/10.1016/j.nbd.2021.105303. 


\section{INPP5D expression is associated with risk for Alzheimer's disease and induced by}

2 plaque-associated microglia

3 Andy P. Tsai ${ }^{1 \#}$, Peter Bor-Chian Lin ${ }^{1 \#}$, Chuanpeng Dong ${ }^{2 \#}$, Miguel Moutinho ${ }^{1}$, Brad T. Casali $^{1.3}$,

4 Yunlong Liu ${ }^{2}$, Bruce T. Lamb ${ }^{1,4}$, Gary E. Landreth ${ }^{1,5}$, Adrian L. Oblak ${ }^{1,6^{*}}$, Kwangsik Nho $^{6^{*}}$

5 1. Stark Neurosciences Research Institute, IUSM, Indianapolis, IN, USA. tandy@iu.edu (A.P.T);

6 pblin@iu.edu (P.B.L); mmoutinh@iu.edu (M.M); glandret@iu.edu (G.E.L); btlamb@iu.edu

7 (B.T.L); aoblak@iupui.edu (A.L.O)

8 2. Department of Medical and Molecular Genetics, Center for Computational Biology and

9 Bioinformatics, IUSM, Indianapolis, IN, USA. cpdong@iu.edu (C.D); yunliu@iu.edu (Y.L)

10 3. Department of Neurosciences, Case Western Reserve University, School of Medicine,

11 Cleveland, OH, USA. btc8@case.edu (B.T.C).

12 4. Department of Medical and Molecular Genetics, IUSM, Indianapolis, IN, USA. btlamb@iu.edu

13 (B.T.L)

14 5. Department of Anatomy and Cell Biology, IUSM, Indianapolis, IN, USA. glandret@iu.edu 15 (G.E.L)

$16{ }^{6 .}$ Department of Radiology \& Imaging Sciences, IUSM, Indianapolis, IN, USA.

17 aoblak@iupui.edu (A.L.O); knho@iupui.edu (K.N)

18 Corresponding Author: *For correspondence contact

19 Adrian L. Oblak, Department of Radiology \& Imaging Sciences, IUSM, Indianapolis, IN, USA,

20 Methodist GH4101, RADY, Indianapolis, IN, 46202, USA. Phone: +1-317-274-0107; Email:

\section{1 aoblak@iupui.edu}

22 Kwangsik Nho, Department of Radiology \& Imaging Sciences, IUSM, Indianapolis, IN, USA,

23 Methodist GH4101, RADY, Indianapolis, IN, 46202, USA. Phone: +1-317-963-7503; Email:

\section{4 knho@iupui.edu}

$25 *$ Corresponding author

26 \#Contributed equally 


\section{$27 \quad$ Abstract}

\section{Background}

Alzheimer's disease (AD) is a progressive neurodegenerative disorder characterized by

30 cognitive decline, robust microgliosis, neuroinflammation, and neuronal loss. Genome-wide

31 association studies recently highlighted a prominent role for microglia in late-onset AD (LOAD).

32 Specifically, inositol polyphosphate-5-phosphatase (INPP5D), also known as SHIP1, is

33 selectively expressed in brain microglia and has been reported to be associated with LOAD.

34 Although INPP5D is likely a crucial player in AD pathophysiology, its role in disease onset and

35 progression remains unclear.

\section{Methods}

37 We performed differential gene expression analysis to investigate INPP5D expression in

38 LOAD and its association with plaque density and microglial markers using transcriptomic

39 (RNA-Seq) data from the Accelerating Medicines Partnership for Alzheimer's Disease (AMP-

40 AD) cohort. We also performed quantitative real-time PCR, immunoblotting, and

41 immunofluorescence assays to assess INPP5D expression in the 5xFAD amyloid mouse model.

\section{Results}

43 Differential gene expression analysis found that INPP5D expression was upregulated in

44 LOAD and positively correlated with amyloid plaque density. In addition, in 5xFAD mice, Inpp5d

45 expression increased as the disease progressed, and selectively in plaque-associated

46 microglia. Increased Inpp5d expression levels in 5xFAD mice were abolished entirely by

47 depleting microglia with the colony-stimulating factor receptor-1 antagonist PLX5622.

\section{Conclusions}

Our findings show that INPP5D expression increases as AD progresses, predominantly

50 in plaque-associated microglia. Importantly, we provide the first evidence that increased

51 INPP5D expression might be a risk factor in AD, highlighting INPP5D as a potential therapeutic 
52 target. Moreover, we have shown that the 5xFAD mouse model is appropriate for studying 53 INPP5D in AD.

Keywords: Alzheimer's disease (AD), Microglia, INPP5D, AD risk, Plaque

\section{Background}

Alzheimer's disease $(A D)$ is the most common cause of dementia, with pathogenesis arising from perturbed $\beta$-amyloid $(A \beta)$ homeostasis in the brain [1]. The mechanisms underlying

60 the development of the most common form of AD, late-onset AD (LOAD), are still unknown.

61 Microglia, the primary immune cells in the brain play a crucial role in AD pathogenesis [2].

62 Recent large-scale genome-wide association studies (GWAS) reported that many genetic loci

63 associated with LOAD risk are related to inflammatory pathways, suggesting that microglia are

64 involved in modulating AD pathogenesis [3,4]. Among the microglia-related genetic factors in

65 LOAD, a common variant in INPP5D (phosphatidylinositol 3,4,5-trisphosphate 5-phosphatase

66 1), rs35349669, confers an increase in LOAD risk $(O R=1.08)[4,5]$. Conversely, the intronic

67 INPP5D variant rs61068452 is associated with a reduced CSF t-tau/A 1 1-42 ratio, plays a

68 protective role in LOAD ( $p=1.48 \mathrm{E}-07)$ [6]. INPP5D encodes inositol polyphosphate-5-

69 phosphatase which participates in regulation of microglial gene expression [7]. Specifically,

70 INPP5D inhibits signal transduction initiated by activation of immune cell surface receptors,

71 including Triggering receptor expressed on myeloid cells 2 (TREM2), Fc gamma receptor

$72(\mathrm{Fc} \gamma \mathrm{R})$ and Dectin-1 [8]. The conversion of $\mathrm{PI}(3,4,5) \mathrm{P} 3$ to $\mathrm{PI}(3,4) \mathrm{P} 2$ is catalyzed by INPP5D

73 following its translocation from the cytosol to the cytoplasmic membrane. The loss of

$74 \mathrm{PI}(3,4,5) \mathrm{P} 3$ prevents the activation of the immune cell surface receptors [9]. Interestingly,

75 genetic variants of TREM2, Fc $\gamma \mathrm{R}$, and Dectin-1 are also associated with increased AD risk [10-

76 12] and are potentially involved in regulating INPP5D activity. Inhibiting INPP5D promotes 
77 microglial proliferation, phagocytosis, and increases lysosomal compartment size [13]. Although

78 INPP5D has been shown to play an important role in microglial function, its role in AD remains

79 unclear.

Here, we report that INPP5D is upregulated in LOAD, and elevated INPP5D expression

81 levels are associated with microglial markers and amyloid plaque density. Furthermore, in the

82 5xFAD mouse model, we found a disease-progression-dependent increase in INPP5D

83 expression in plaque-associated microglia. Our results suggest that INPP5D plays a role in

84 microglia phenotypes in AD and is a potential target for microglia-focused AD therapies.

\section{Methods}

86 Human participants and RNA-Seq

RNA-Seq data were obtained from the AMP-AD Consortium, including participants of the

88 Mayo Clinic Brain Bank cohort, the Mount Sinai Medical Center Brain Bank (MSBB) cohort, and

89 the Religious Orders Study and Memory and Aging Project (ROSMAP) cohort.

In the Mayo Clinic RNA-Seq dataset [14], the RNA-Seq-based whole transcriptome data

91 were generated from human samples of 151 temporal cortices (TCX) ( 71 cognitively normal

92 older adult controls (CN) and 80 LOAD) and 151 cerebella (CER) (72 CN and 79 LOAD). LOAD

93 participants met the neuropathological criteria for $A D$ (Braak score $\geq 4.0$ ), and cognitively normal

94 participants had no neurodegenerative diagnosis (Braak score $\leq 3.0$ ).

95 In the MSBB dataset [15], data were generated from human samples from CN, mild

96 cognitive impairment (MCl), and LOAD participants' parahippocampal gyrus (PHG) and inferior

97 frontal gyrus (IFG), superior temporal gyrus (STG) and frontal pole (FP). The clinical dementia

98 rating scale (CDR) was used to assess dementia and cognitive status [16]. LOAD patients had a

99 CDR $\geq 0.5$, while $\mathrm{MCl}$ and $\mathrm{CN}$ participants had a CDR of 0.5 and 0 , respectively. CN participants

100 had no significant memory concerns. This study included 108 participants $(16 \mathrm{CN}, 14 \mathrm{MCl}$, and 
78 LOAD) for PHG, 137 participants (21 CN, $18 \mathrm{MCl}$, and 98 LOAD) for STG, 136 participants (18 CN, $16 \mathrm{MCl}$, and $102 \mathrm{LOAD}$ ) for IFG, and 153 participants (22 CN, $20 \mathrm{MCl}$, and $111 \mathrm{LOAD}$ ) 103 for FP. prefrontal cortices of 241 participants ( $86 \mathrm{CN}$ and $155 \mathrm{LOAD}$ ).

\section{Animal models}

Wild-type (WT) and 5xFAD mice were maintained on the C57BL/6J background (JAX MMRRC Stock\# 034848) for IHC and qPCR studies. Two-, four-, six-, eight-, and twelve-month-old mice were used. In the PLX5622 study, we used WT and 5XFAD mice maintained on the mixed C57BL/6J and SJL background [B6SJL-Tg (APPSwFILon, PSEN1*M146L*L286V) 6799Vas,

111 Stock \#34840-JAX]) (Fig. 3e and 3f). The 5XFAD transgenic mice overexpress five FAD 112 mutations: the APP (695) transgene contains the Swedish (K670N, M671L), Florida (I716V), 113 and London (V717I) mutations and the PSEN1 transgene contains the M146L and L286V FAD

114 mutations. Up to five mice were housed per cage with SaniChip bedding and

115 LabDiet $₫ 5$ K52/5K67 (6\% fat) feed. The colony room was kept on a 12:12 hr. light/dark

116 schedule with the lights on from 7:00 am to 7:00 pm daily. They were bred and housed in 117 specific-pathogen-free conditions. Both male and female mice were used.

\section{PLX5622 animal treatment}

At four months of age, either normal rodent diet or PLX5622-containing chow was

120 administered to 5XFAD mice for 28 days. An additional cohort of four-month-old mice was

121 treated with PLX5622 or control diet for 28 days, then discontinued from PLX5622 feed and fed

122 a normal rodent diet for an additional 28 days. At six months of age, this cohort of mice was

123 euthanized. Plexxikon Inc. provided PLX5622 formulated in AIN-7 diet at 1200 mg/kg [18].

\section{Statistical analysis}


In the human study, differential expression analysis was performed using limma software

126 [19] to investigate the diagnosis group difference of INPP5D between CN, MCI, and LOAD. Age, 127 sex, and APOE \&4 carrier status were used as covariates. To investigate the association

128 between INPP5D expression levels and amyloid plaque density or expression levels of

129 microglia-specific markers (AIF1 and TMEM119), we used a generalized linear regression

130 model with INPP5D expression levels as a dependent variable and plaque density or microglia-

131 specific markers along with age, sex, and $A P O E \varepsilon 4$ carrier status as explanatory variables. The

132 regression was performed with the "glm" function from the stats package in R (version 3.6.1).

133 In the mouse study, GraphPad Prism (Version 8.4.3) was used to perform the statistical

134 analyses. Differential expression analysis of both gene and protein levels between WT and

135 5xFAD mice was performed using unpaired Student's t-test. The statistical comparisons

136 between mice with and without PLX5622 treatments were performed with one-way ANOVA

137 followed by Tukey's posthoc test. Graphs represent the mean and standard error of the mean.

\section{RNA extraction and quantitative real-time PCR}

Mice were anesthetized with Avertin and perfused with ice-cold phosphate-buffered

140 saline (PBS). The cortical and hippocampal regions from the hemisphere were micro-dissected

141 and stored at $-80^{\circ} \mathrm{C}$. Frozen brain tissue was homogenized in buffer containing $20 \mathrm{mM}$ Tris- $\mathrm{HCl}$

142 (pH=7.4), $250 \mathrm{mM}$ sucrose, $0.5 \mathrm{mM}$ EGTA, $0.5 \mathrm{mM}$ EDTA, RNase-free water, and stored in an

143 equal volume of RNA-Bee (Amsbio, CS-104B) at $-80^{\circ} \mathrm{C}$ until RNA extraction. RNA was isolated

144 by chloroform extraction and purified with the Purelink RNA Mini Kit (Life Technologies

145 \#12183020) with an on-column DNAse Purelink Lit (Life Technologies \#12183025). 500 ng RNA

146 was converted to cDNA with the High-Capacity RNA-to-cDNA Kit (Applied Biosystems

$147 \quad$ \#4388950), and qPCR was performed on a StepOne Plus Real-Time PCR system (Life

148 Technologies). Relative gene expression was determined with the $2^{-\triangle \Delta C T}$ method and assessed

149 relative to Gapdh (Mm99999915_g1). Inpp5d primer: Taqman Gene Expression Assay (Inpp5d: 
Mm00494987_m1 from the Life Technologies). Student's $t$-test was performed for qPCR

151 assays, comparing WT with 5xFAD animals.

\section{Immunofluorescence}

Brains were fixed in $4 \%$ PFA overnight at $4^{\circ} \mathrm{C}$. Following overnight fixation, brains were

154 cryoprotected in $30 \%$ sucrose at $4^{\circ} \mathrm{C}$ and embedded. Brains were processed on a microtome as

$15530 \mu \mathrm{m}$ free-floating sections. For immunostaining, at least three matched brain sections were

156 used. Free-floating sections were washed and permeabilized in $0.1 \%$ Triton in PBS (PBST),

157 followed by antigen retrieval using 1x Reveal Decloaker (Biocare Medical) at $85^{\circ} \mathrm{C}$ for 10 mins.

158 Sections were blocked in 5\% normal donkey serum in PBST for $1 \mathrm{hr}$. at room temperature (RT).

159 The following primary antibodies were incubated in 5\% normal donkey serum in PBST overnight

160 at $4^{\circ} \mathrm{C}$ : IBA1 (Novus Biologicals \#NB100-1028 in goat, 1:1000); $6 \mathrm{E} 10$ (BioLegend \#803001 in

161 mouse, 1:1000; AB_2564653); and SHIP1/INPP5D (Cell Signaling Technology (CST) \#4C8,

162 1:500, Rabbit mAb provided by CST in collaboration with Dr. Richard W. Cho). Sections were

163 washed and visualized using respective species-specific AlexaFluor fluorescent antibodies

164 (diluted 1:1000 in 5\% normal donkey serum in PBST for $1 \mathrm{hr}$. at RT). Sections were

165 counterstained and mounted onto slides. For X-34 staining (Sigma, \#SML1954), sections were

166 dried at RT, rehydrated in PBST, and stained for ten mins at RT. Sections were then washed

167 five times in double-distilled water and washed again in PBST for five mins. Images were

168 acquired on a fluorescent microscope with similar exposure and gains across stains and

169 animals. Images were merged using ImageJ (NIH).

\section{Immunoblotting}

Tissue was extracted and processed as described above, then centrifuged. Protein

172 concentration was measured with a BCA kit (Thermo Scientific). $50 \mu \mathrm{g}$ of protein per sample

173 was boiled in SDS-PAGE protein sample buffer for 10 mins at $95^{\circ} \mathrm{C}$, loaded into $4-12 \%$ Bis-Tris 
174 gels (Life Technologies) and run at $100 \mathrm{~V}$ for 90 mins. The following primer antibodies were

175 used: SHIP1/INPP5D (CST \#4C8 1:500, Rabbit mAb) and GAPDH (Santa Cruz \#sc-32233).

176 Each sample was normalized to GAPDH, and the graphs represent the values normalized to the 177 mean of the WT mice group at each time point.

\section{$178 \quad \underline{\text { Results }}$}

179 INPP5D expression levels are increased in LOAD.

INPP5D is a member of the inositol polyphosphate-5-phosphatase (INPP5) family and possesses a set of core domains, including an N-terminal SH2 domain (amino acids 5-101),

182 Pleckstrin homology-related (PH-R) domain (amino acids 292-401), lipid phosphatase region 183 (amino acids 401-866) with C2 domain (amino acids 725-863), and C-terminal proline-rich 184 region (amino acids 920-1148) with two SH3 domains (amino acids 969-974 and 1040-1051)

185 (Fig. 1a). Differential expression analysis was performed using RNA-Seq data from seven brain 186 regions from the AMP-AD cohort. Expression levels of INPP5D were increased in the temporal cortex $(\log F C=0.35, p=1.12 E-02 ;$ Fig. 1b), parahippocampal gyrus $(\log F C=0.54, p=7.17 E-03$;

188 Fig. 1c), and inferior frontal gyrus ( $\log F C=0.44, p=2.33 E-03 ;$ Fig. 1d) of LOAD patients with age and sex as covariates (Table 1). Interestingly, INPP5D expression was also found to be increased in the inferior frontal gyrus of LOAD patients compared with $\mathrm{MCl}$ subjects

$191 \quad(\log F C=0.45, p=6.76 E-03 ;$ Fig. 1d). Results were similar when $A P O E \varepsilon 4$ carrier status was used 192 as an additional covariate. INPP5D remained overexpressed in the temporal cortex $193(\log F C=0.34, p=2.75 E-02)$, parahippocampal gyrus $(\log F C=0.53, p=1.08 E-02)$, and inferior 194 frontal gyrus ( $\log F C=0.42, p=4.35 E-03)$ of $L O A D$ patients. However, we did not find any 195 differences between the diagnosis groups in the cerebellum, frontal pole, superior temporal 196 gyrus, or dorsolateral prefrontal cortex (Table 1). To examine whether INPP5D was associated 197 with microglia, we analyzed the association between INPP5D and microglia-specific marker 198 genes (AIF1 and TMEM119). AIF1 and TMEM119 were significantly associated with INPP5D 
expression levels in the parahippocampal gyrus (AIF1: $\beta=0.4386, p=4.10 E-07 ;$ TMEM119: $\beta=0.7647, p=<2 E-16)$, inferior frontal gyrus (AIF1: $\beta=0.2862, p=6.36 E-08 ;$ TMEM119: $\beta=0.6109$, $p=<2 E-16)$, frontal pole (AIF1: $\beta=0.2179, p=4.53 E-04 ;$ TMEM119 $\beta=0.5062, p=4.00 E-15)$, and superior temporal gyrus (AIF1: $\beta=0.3013, p=5.36 \mathrm{E}-07$; TMEM119: $\beta=0.6914, p=<2 \mathrm{E}-16)$ (Table 2).

INPP5D expression levels are associated with amyloid plaque density in the human 205 brain.

We investigated the association between INPP5D expression levels and mean amyloid plaque densities in four brain regions (Table 2). Expression levels of INPP5D were associated with amyloid plaques in the parahippocampal gyrus $(\beta=0.0212, p=3.02 E-03$; Fig. 2a), inferior frontal gyrus $(\beta=0.0163, p=1.95 E-03$; Fig. 2b), frontal pole $(\beta=0.0151, p=1.22 E-02 ;$ Fig. 2c $)$, and superior temporal gyrus $(\beta=0.0220, p=5.05 E-04 ;$ Fig. $2 d)$.

We recapitulated our findings from the human data in the amyloidogenic mouse model,

213 5xFAD. We observed increased Inpp5d mRNA levels in 5xFAD mice throughout disease 214 progression compared with WT controls in the brain cortex (Fig. 3a) and hippocampus (Fig. 3b) 215 of four-, six-, eight-, and twelve-month-old mice (4-months: 1.57-fold in the cortex, 1.40-fold in 216 the hippocampus; 6-months: 1.86-fold in the cortex, 2.61-fold in the hippocampus; 8-months: 217 2.23-fold in the cortex and 2.53-fold in the hippocampus; and 12-months: 1.93-fold in the cortex 218 and 2.16-fold in the hippocampus). Similarly, INPP5D protein levels were increased in the 219 cortex of 5xFAD mice at four and eight months of age ( 1.79 and 3.31 -fold, respectively; $p=0.06)$ 220 (Fig. 3c and 3d). To assess Inpp5d induction was dependent on microglia, we depleted 221 microglia in four-month-old 5xFAD mice by treating the animals with the colony-stimulating 222 factor receptor-1 antagonist PLX5622 (PLX) for 28 days [18]. PLX treatment completely 223 abolished the increase of Inpp5d in 5xFAD mice (Fig. 3e). Furthermore, expression levels of 
224 Inpp5d were restored after switching from the PLX diet to a normal diet for 28 further days (Fig.

$2253 f)$.

226 INPP5D expression levels are increased in plaque-associated microglia Immunohistochemistry of 5xFAD mice brain slices at eight months old revealed that

228 Inpp5d was mainly expressed in plaque-associated microglia (Fig. 4). INPP5D- and IBA1

229 (AIF1)-positive microglia cluster around 6E10-positive or X-34-positive plaques in the cortex

230 (Fig. 4a) and subiculum (Fig. 4b). We did not detect any INPP5D expression in WT control mice

231 (data not shown). Furthermore, analysis of transcriptomic data of sorted microglia from WT

232 mouse cortex-injected labeled apoptotic neurons [12] revealed a reduction of Inpp5d expression

233 levels in phagocytic microglia compared with non-phagocytic microglia (Fig. 4c), which is in

234 agreement with the report that INPP5D inhibition promotes microglial phagocytosis [13].

\section{Discussion}

236 Although genetic variants in INPP5D have been associated with LOAD risk [5, 6, 20, 21],

237 the role of INPP5D in AD remains unclear. We identified that INPP5D expression levels are

238 increased in the brain of LOAD patients. Furthermore, expression levels of INPP5D positively

239 correlate with brain amyloid plaque density and AIF1 and TMEM119 (microglial marker gene)

240 expression [22-24]. We observed similar findings in the 5xFAD amyloidogenic model, which

241 exhibited an increase in gene and protein expression levels of Inpp5d with disease progression,

242 predominately in plaque-associated microglia, suggesting induction of Inpp $5 d$ in plaque-

243 proximal microglia. Similarly, a recent study reported that Inpp5d is strongly correlated with

244 amyloid plaque deposition in the APPPS1 mouse model $[25,26]$. These findings are consistent

245 with the observation of microgliosis in both $A D$ and its mouse models.

INPP5D inhibition has been associated with microglial activation and increased

247 phagocytic activity, which is consistent with our transcriptomic data of sorted microglia from 
248 murine brains injected with apoptotic neurons [12], showing a decrease in Inpp5d expression in

249 phagocytic microglia compared to non-phagocytic. These findings support the hypothesis that

250 an increase in INPP5D expression in AD is a part of an endogenous homeostatic microglial

251 response to negatively control their own activity. However, this "brake" might be excessive in

$252 \mathrm{AD}$, as reflected in our findings that INPP5D expression is elevated in LOAD. INPP5D

253 overexpression might result in microglia with deficient phagocytic capacity, resulting in

254 increased $A \beta$ deposition and neurodegeneration. Thus, the pharmacological targeting of

255 INPP5D might be a novel therapeutic strategy to shift microglia towards a beneficial phenotype

256 in AD. Future studies in genetic mouse models are necessary to further clarify the role of

257 INPP5D in microglial function and AD progression.

\section{Conclusions}

259 In conclusion, our results demonstrate that INPP5D plays a crucial role in AD

260 pathophysiology and is a potential therapeutic target. INPP5D expression is upregulated in

261 LOAD and positively correlated with amyloid plaque density. Inpp5d expression increases in the

262 microglia of 5xFAD mice as AD progresses, predominately in plaque-associated microglia.

263 Future studies investigating the effect of INPP5D loss-of-function on microglial phenotypes and

264 AD progression may allow for the development of microglial-targeted AD therapies.

\section{List of abbreviations}

266

AD: Alzheimer's disease, LOAD: late-onset AD, GWAS: genome-wide association

267 studies, INPP5D: phosphatidylinositol 3,4,5-trisphosphate 5-phosphatase 1, PI(3,4,5)P3:

268 phosphatidylinositol (3,4,5)-trisphosphate, $\mathrm{PI}(3,4) \mathrm{P} 2$ : phosphatidylinositol $(3,4)$-bisphosphate,

269 CSF: cerebrospinal fluid, OR: odds ratio, Cl: confidence interval, $\beta$ : $\beta$ coefficient, WT: wild-type,

$270 \mathrm{MCl}$ : mild cognitive impairment, APOE $\varepsilon 4$ : apolipoprotein $\varepsilon 4$ allele, PFA: paraformaldehyde, 
271 PCR: polymerase chain reaction, Seq: sequencing, ANOVA: analysis of variance, qPCR:

272 quantitative real-time PCR, mAb: monoclonal antibody.

\section{Declarations}

274 Ethics approval and consent to participate

$275 \quad$ Animals used in the study were housed in the Stark Neurosciences Research Institute

276 Laboratory Animal Resource Center at Indiana University School of Medicine and all

277 experimental procedures were approved by the Institutional Animal Care and Use Committee.

278 Consent for publication

$279 \quad$ All participants were properly consented for this study.

280 Availability of data and materials

281 The datasets analyzed during the current study are available from the corresponding 282 author on reasonable request.

283 Competing interests

$284 \quad$ The authors declare that they have no competing interests.

285 Funding

286 This work was supported by NIA grant RF1 AG051495 (B.T.L and G.E.L), NIA grant RF1

287 AG050597 (G.E.L), NIA grant U54 AG054345 (B.T.L et al.), NIA grant K01 AG054753 (A.L.O),

288 NIA grant R03 AG063250 (K.N), and NIH grant NLM R01 LM012535 (K.N)

289 Author contributions

290 A.P.T, P.B.L, C.D, Y.L, B.T.L, G.E.L, A.L.O, and K.N designed the study. A.P.T, P.B.L, 291 C.D, M.M, B.T.C, and K.N performed the experiments and analyzed the data. A.P.T, M.M, 
292 G.E.L, A.L.O, and K.N wrote the manuscript. All authors discussed the results and commented 293 on the manuscript.

\section{Acknowledgments}

We would like to thank Dr. Richard W. Cho at Cell Signaling Technology for providing SHIP1/INPP5D Rabbit mAb. We thank Louise Pay for critical comments on the manuscript. We

297 thank Cynthia M. Ingraham, Deborah D. Baker, Christopher D. Lloyd, Stephanie J. Bissel, 298 Shweta S. Puntambekar, Guixiang Xu, Roxanne Y. Williams, and Teaya N. Thomas for their 299 help with taking care of the mice, genotyping, and helpful discussions. We thank the 300 International Genomics of Alzheimer's Project (IGAP) for providing summary results data for 301 these analyses. IGAP investigators contributed to the design and implementation of IGAP 302 and/or provided data but did not participate in analysis or writing of this report. IGAP was made possible by the generous participation of the control subjects, the patients, and their families.

304 The i-Select chips were funded by the French National Foundation on Alzheimer's disease and related disorders. EADI was supported by the LABEX (laboratory of excellence program investment for the future) DISTALZ grant, Inserm, Institut Pasteur de Lille, Université de Lille 2,

307 and the Lille University Hospital. GERAD/PERADES was supported by the Medical Research

308 Council (Grant $n^{\circ}$ 503480), Alzheimer's Research UK (Grant $n^{\circ}$ 503176), the Wellcome Trust

309 (Grant $n^{\circ}$ 082604/2/07/Z) and German Federal Ministry of Education and Research (BMBF):

310 Competence Network Dementia (CND) grant n 01GI0102, 01GI0711, 01GI0420. CHARGE was

311 partly supported by the NIH/NIA grant R01 AG033193 and the NIA AG081220 and AGES

312 contract N01-AG-12100, the NHLBI grant R01 HL105756, the Icelandic Heart Association, and

313 the Erasmus Medical Center and Erasmus University. ADGC was supported by the NIH/NIA

314 grants: U01 AG032984, U24 AG021886, U01 AG016976, and the Alzheimer's Association grant 315 ADGC-10-196728. 
318 1. Lee CY, Landreth GE: The role of microglia in amyloid clearance from the AD brain. 319 J Neural Transm (Vienna) 2010, 117(8):949-960.

320 2. Mandrekar-Colucci S, Landreth GE: Microglia and inflammation in Alzheimer's 321 disease. CNS Neurol Disord Drug Targets 2010, 9(2):156-167.

322 3. Karch CM, Goate AM: Alzheimer's disease risk genes and mechanisms of disease 323 pathogenesis. Biol Psychiatry 2015, 77(1):43-51.

324 4. Lambert JC, Ibrahim-Verbaas CA, Harold D, Naj AC, Sims R, Bellenguez C, DeStafano 325 AL, Bis JC, Beecham GW, Grenier-Boley B et al: Meta-analysis of 74,046 individuals 326 identifies 11 new susceptibility loci for Alzheimer's disease. Nat Genet 2013, 45(12):1452-1458.

5. Jing H, Zhu JX, Wang HF, Zhang W, Zheng ZJ, Kong LL, Tan CC, Wang ZX, Tan L, Tan L: INPP5D rs35349669 polymorphism with late-onset Alzheimer's disease: A replication study and meta-analysis. Oncotarget 2016, 7(43):69225-69230.

6. Yao X, Risacher SL, Nho K, Saykin AJ, Wang Z, Shen L, Alzheimer's Disease Neuroimaging I: Targeted genetic analysis of cerebral blood flow imaging phenotypes implicates the INPP5D gene. Neurobiol Aging 2019, 81:213-221.

334 7. Viernes DR, Choi LB, Kerr WG, Chisholm JD: Discovery and development of small molecule SHIP phosphatase modulators. Med Res Rev 2014, 34(4):795-824.

8. Peng Q, Malhotra S, Torchia JA, Kerr WG, Coggeshall KM, Humphrey MB: TREM2- and DAP12-dependent activation of PI3K requires DAP10 and is inhibited by SHIP1. Sci Signal 2010, 3(122):ra38.

9. Rohrschneider LR, Fuller JF, Wolf I, Liu Y, Lucas DM: Structure, function, and biology of SHIP proteins. Genes Dev 2000, 14(5):505-520. 
341 10. Sims R, van der Lee SJ, Naj AC, Bellenguez C, Badarinarayan N, Jakobsdottir J, Kunkle BW, Boland A, Raybould R, Bis JC et al: Rare coding variants in PLCG2, ABI3, and TREM2 implicate microglial-mediated innate immunity in Alzheimer's disease. Nat Genet 2017, 49(9):1373-1384.

345 11. Tsai AP, Dong C, Preuss C, Moutinho M, Lin PB-C, Hajicek N, Sondek J, Bissel SJ, Oblak AL, Carter GW et al: <em>PLCG2</em> as a Risk Factor for Alzheimer's Disease. bioRxiv 2020:2020.2005.2019.104216.

348 12. Krasemann S, Madore C, Cialic R, Baufeld C, Calcagno N, El Fatimy R, Beckers L, O'Loughlin E, Xu Y, Fanek Z et al: The TREM2-APOE Pathway Drives the Transcriptional Phenotype of Dysfunctional Microglia in Neurodegenerative Diseases. Immunity 2017, 47(3):566-581 e569.

13. Pedicone C, Fernandes S, Dungan OM, Dormann SM, Viernes DR, Adhikari AA, Choi LB, De Jong EP, Chisholm JD, Kerr WG: Pan-SHIP1/2 inhibitors promote microglia effector functions essential for CNS homeostasis. J Cell Sci 2020, 133(5).

14. Allen M, Carrasquillo MM, Funk C, Heavner BD, Zou F, Younkin CS, Burgess JD, Chai HS, Crook J, Eddy JA et al: Human whole genome genotype and transcriptome data

15. Wang M, Beckmann ND, Roussos P, Wang E, Zhou X, Wang Q, Ming C, Neff R, Ma W, Fullard JF et al: The Mount Sinai cohort of large-scale genomic, transcriptomic and proteomic data in Alzheimer's disease. Sci Data 2018, 5:180185.

363 17. Bennett DA, Buchman AS, Boyle PA, Barnes LL, Wilson RS, Schneider JA: Religious 64(s1):S161-S189. 
18. Casali BT, MacPherson KP, Reed-Geaghan EG, Landreth GE: Microglia depletion rapidly and reversibly alters amyloid pathology by modification of plaque compaction and morphologies. Neurobiol Dis 2020, 142:104956.

19. Ritchie ME, Phipson B, Wu D, Hu Y, Law CW, Shi W, Smyth GK: limma powers differential expression analyses for RNA-sequencing and microarray studies. Nucleic Acids Res 2015, 43(7):e47.

20. Farfel JM, Yu L, Buchman AS, Schneider JA, De Jager PL, Bennett DA: Relation of genomic variants for Alzheimer disease dementia to common neuropathologies. Neurology 2016, 87(5):489-496.

22. Hopperton KE, Mohammad D, Trepanier MO, Giuliano V, Bazinet RP: Markers of

23. Kaiser T, Feng G: Tmem119-EGFP and Tmem119-CreERT2 Transgenic Mice for

24. Satoh J, Kino Y, Asahina N, Takitani M, Miyoshi J, Ishida T, Saito Y: TMEM119 marks a

26. Salih DA, Bayram S, Guelfi S, Reynolds RH, Shoai M, Ryten M, Brenton JW, Zhang D,

25. Radde R, Bolmont T, Kaeser SA, Coomaraswamy J, Lindau D, Stoltze L, Calhoun ME, influences Alzheimer's disease risk. Brain Commun 2019, 1(1):fcz022. 
Fig. 1 Relative quantification of INPP5D expression in the studied participants

a

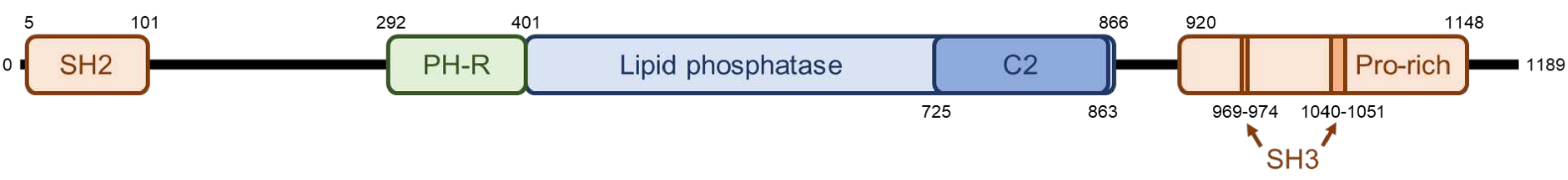

Fig 1. Relative quantification of INPP5D expression in the studied participants

(a) Domain architecture of INPP5D drawn to scale. Gene expression of INPP5D is showed as logCPM values in (b) Temporal cortex (TCX)Mayo, (c) Parahippocampal gyrus (PHG)-MSBB, (d) Inferior frontal gyrus (IFG)-MSBB, (e) Cerebellum (CER)-Mayo, (f) Frontal pole (FP)MSBB, (g) Superior temporal gyrus (STG)-MSBB, (h) Dorsolateral prefrontal cortex (DLPFC)-ROSMAP.

SH2 Src Homology 2 domain, SH3 SRC Homology 3 domain, C2 C2 domain 
Fig. 1 Relative quantification of INPP5D expression in the studied participants

b

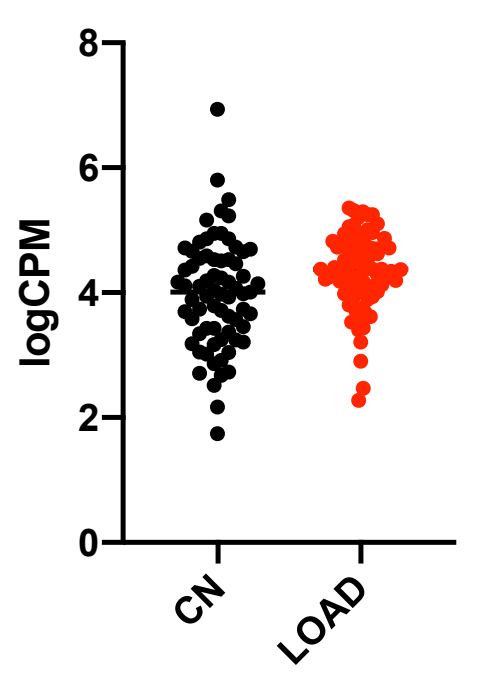

Temporal cortex

e

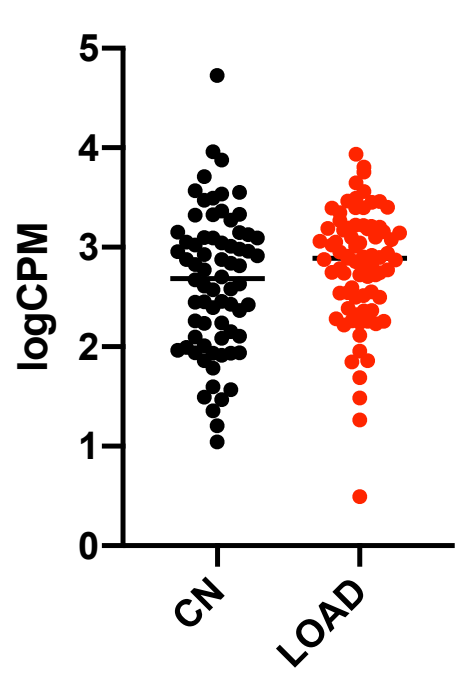

Cerebellum
C

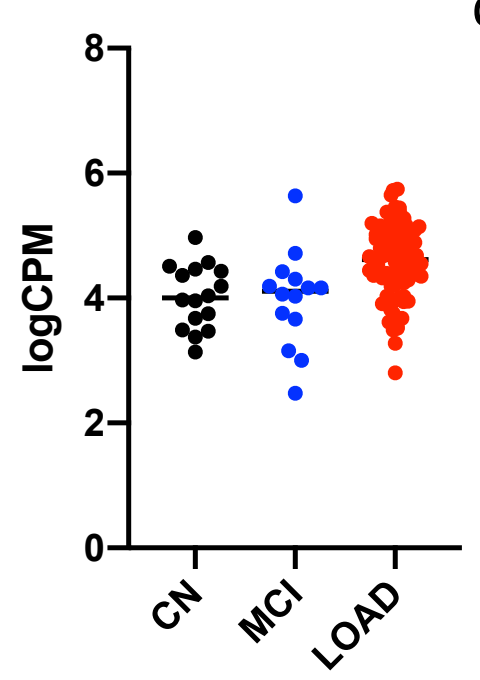

Parahippocampal gyrus

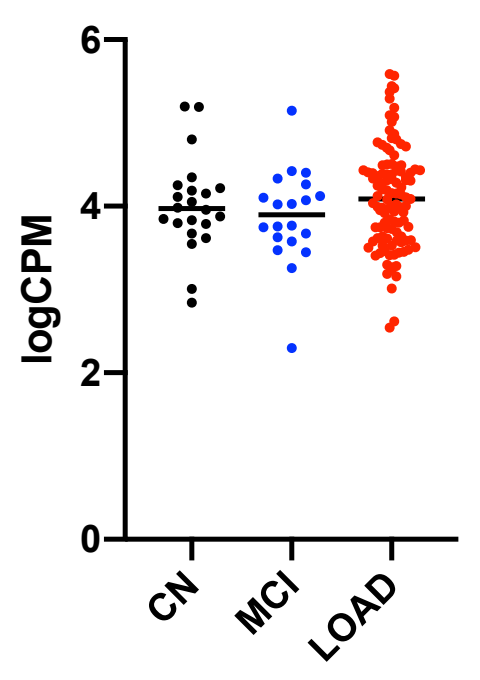

Frontal pole

d

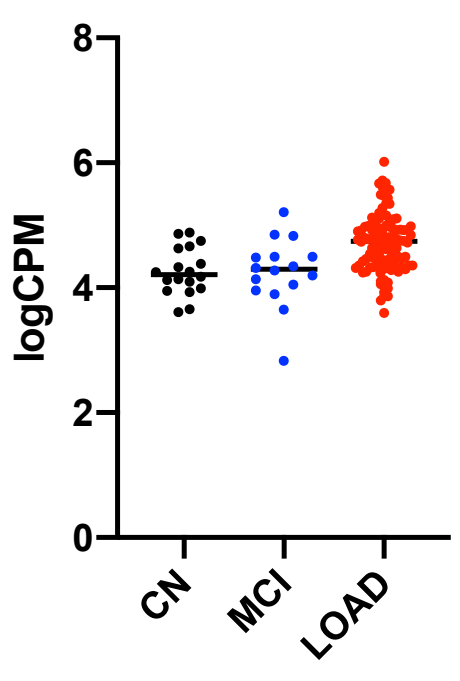

Inferior frontal gyrus

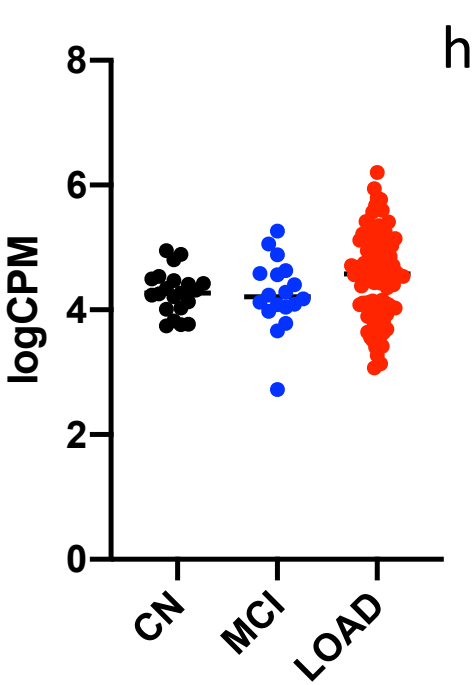

Superior temporal gyrus

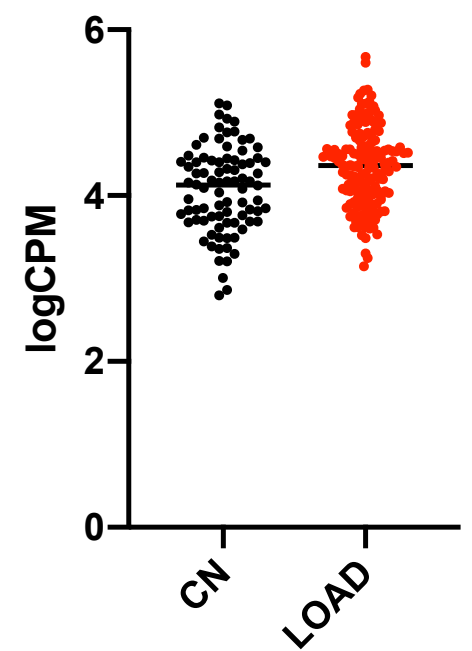

Dorsolateral prefrontal cortex

$\mathrm{CN}$ cognitively normal, $\mathrm{MCl}$ mild cognitive impairment, LOAD Late-Onset Alzheimer's disease 
Table 1.

INPP5D expression levels were increased in LOAD

\begin{tabular}{|c|c|c|c|c|c|c|c|c|}
\hline Brain Regions & Temporal Cortex & \multicolumn{3}{|c|}{ Parahippocampal Gyrus } & \multicolumn{3}{|c|}{ Inferior Frontal Gyrus } & \\
\hline \multicolumn{9}{|c|}{ Covariate: Age and Sex } \\
\hline Contrast & CN vs. LOAD & $\mathrm{CN}$ vs. $\mathrm{MCl}$ & MCI vs. LOAD & $C N$ vs. LOAD & $\mathrm{CN}$ vs. $\mathrm{MCl}$ & $\mathrm{MCl}$ vs. LOAD & CN vs. LOAD & \\
\hline $\log \mathrm{FC}$ & 0.34916854 & 0.012305567 & 0.565288103 & 0.536580956 & -0.009944963 & 0.44545717 & 0.43695423 & \\
\hline p-value & 1.12E-02 & 9.99E-01 & $7.56 \mathrm{E}-02$ & 7.17E-03 & $1.00 \mathrm{E}+00$ & 6.76E-03 & 2.33E-03 & \\
\hline \multicolumn{9}{|c|}{ Covariate: Age, Sex, and APOE $\varepsilon 4$ status } \\
\hline Contrast & CN vs. LOAD & $\mathrm{CN}$ vs. $\mathrm{MCl}$ & $\mathrm{MCl}$ vs. LOAD & CN vs. LOAD & $\mathrm{CN}$ vs. $\mathrm{MCl}$ & $\mathrm{MCl}$ vs. LOAD & CN vs. LOAD & \\
\hline $\log \mathrm{FC}$ & 0.34072691 & -0.034336626 & 0.57213088 & 0.52809965 & -0.0546836 & 0.46476734 & 0.42180431 & \\
\hline p-value & 2.75E-02 & $1.00 \mathrm{E}+00$ & $1.00 \mathrm{E}-01$ & $1.08 \mathrm{E}-02$ & $1.00 \mathrm{E}+00$ & 8.00E-03 & 4.35E-03 & \\
\hline Brain Regions & Cerebellum & \multicolumn{3}{|c|}{ Frontal Pole } & \multicolumn{3}{|c|}{ Superior Temporal Gyrus } & Dosolateral Prefrontal Cortex \\
\hline \multicolumn{9}{|c|}{ Covariate: Age and Sex } \\
\hline Contrast & CN vs. LOAD & $\mathrm{CN}$ vs. $\mathrm{MCl}$ & MCI vs. LOAD & CN vs. LOAD & $\mathrm{CN}$ vs. $\mathrm{MCl}$ & $\mathrm{MCl}$ vs. LOAD & CN vs. LOAD & CN vs. LOAD \\
\hline $\log F C$ & 0.15322052 & -0.1498388 & 0.20026554 & 0.09349678 & -0.0115078 & 0.31887371 & 0.28945778 & 0.1761858 \\
\hline p-value & 2.15E-01 & $9.85 \mathrm{E}-01$ & $4.80 \mathrm{E}-01$ & 8.65E-01 & $1.00 \mathrm{E}+00$ & 2.09E-01 & $1.78 \mathrm{E}-01$ & $5.76 \mathrm{E}-02$ \\
\hline \multicolumn{9}{|c|}{ Covariate: Age, Sex, and APOE $\varepsilon 4$ status } \\
\hline Contrast & CN vs. LOAD & $\mathrm{CN}$ vs. $\mathrm{MCl}$ & MCI vs. LOAD & CN vs. LOAD & $\mathrm{CN}$ vs. MCl & $\mathrm{MCl}$ vs. LOAD & $C N$ vs. LOAD & CN vs. LOAD \\
\hline $\log F C$ & 0.207875239 & -0.180168831 & 0.20013891 & 0.068420156 & -0.020260737 & 0.282432909 & 0.22080636 & 0.16972131 \\
\hline p-value & 1.23E-01 & 9.53E-01 & 5.13E-01 & $9.25 \mathrm{E}-01$ & $1.00 \mathrm{E}+00$ & $2.89 \mathrm{E}-01$ & $3.26 \mathrm{E}-01$ & $9.46 \mathrm{E}-02$ \\
\hline
\end{tabular}

Table 1 shows the p-values for the gene expression analyses performed with limma using RNA-Seq data from the AMP-AD Consortium.

TCX temporal cortex, PHG parahippocampal gyrus, STG superior temporal gyrus, IFG inferior frontal gyrus, FP frontal pole, CER cerebellum,

DLPFC dorsolateral prefrontal cortex, $\mathrm{CN}$ cognitively normal , $\mathrm{AD}$ Alzheimer's disease, $\mathrm{MCl}$ mild cognitive impairment, logFC log fold-change 


\section{Fig. 2 Association of INPP5D expression with amyloid plaque mean density}

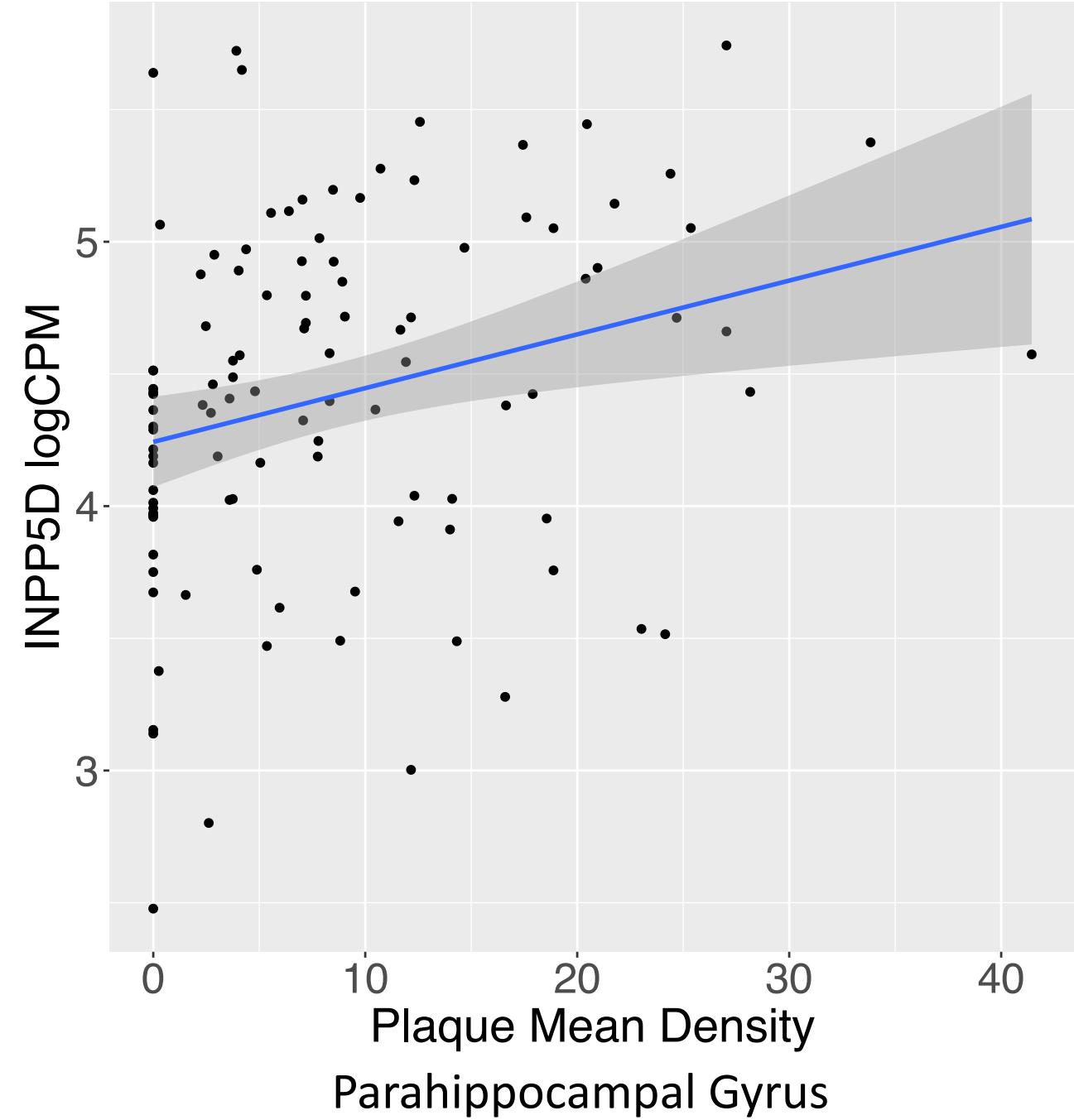

b

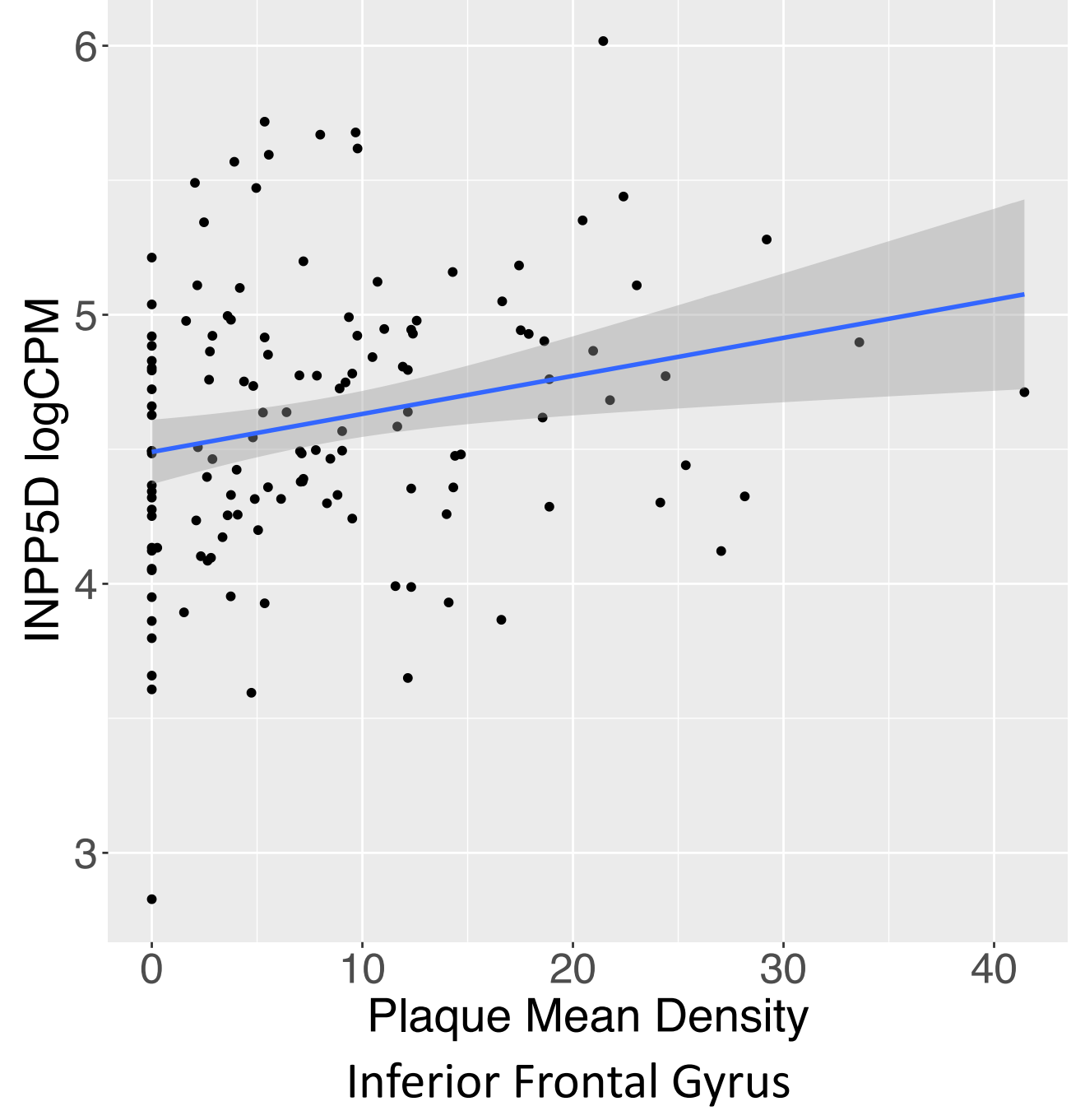




\section{Fig. 2 Association of INPP5D expression with amyloid plaque mean density}
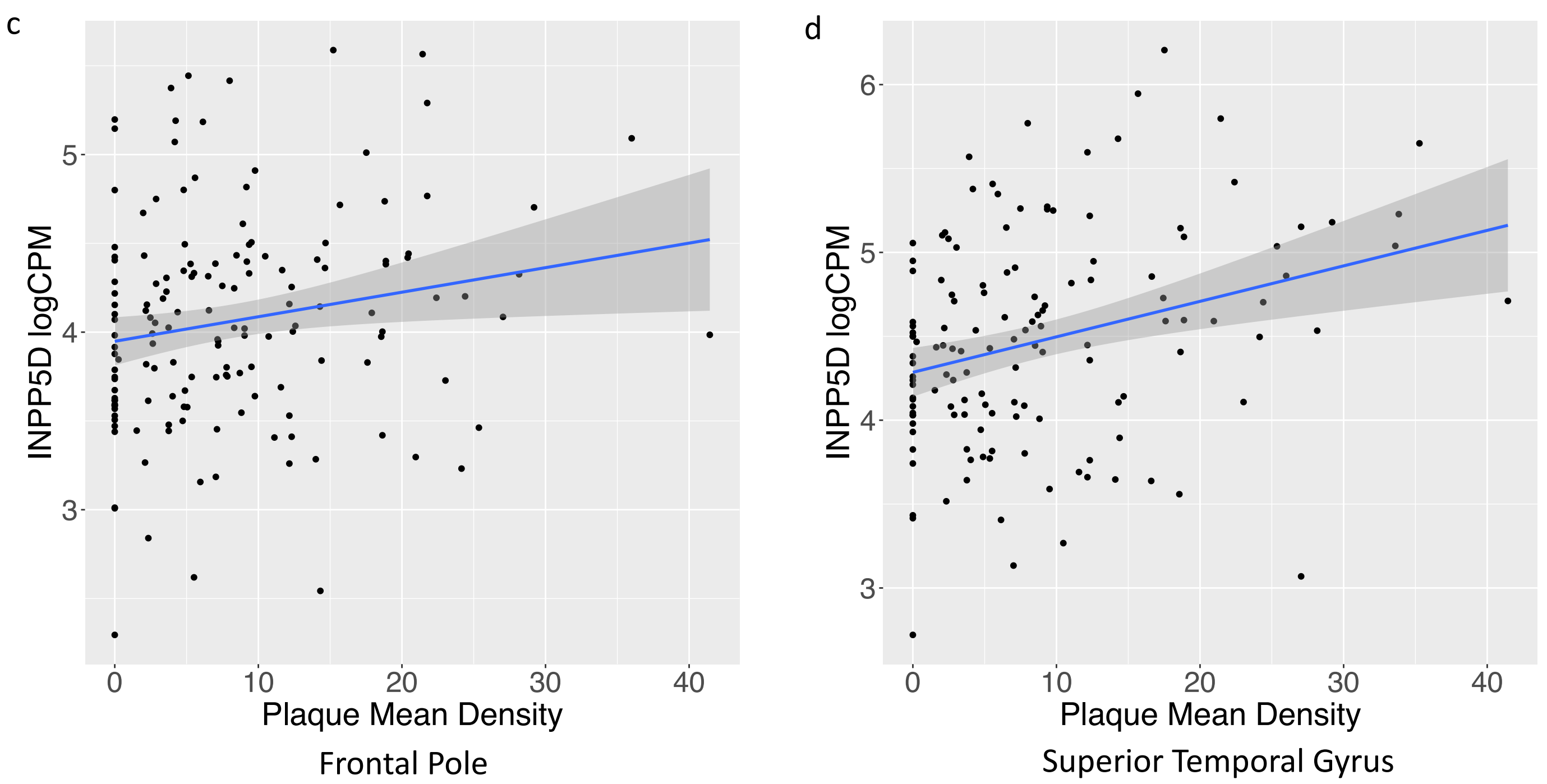

Fig 2. Association of INPP5D expression with amyloid plaque mean density.

The scatter plots show the positive association between INPP5D expression and plaque mean density in (a) parahippocampal gyrus, (b) inferior frontal gyrus, (c) frontal pole, and (d) superior temporal gyrus from the MSBB cohort. 
Table 2.

INPP5D expression levels are associated with amyloid plaque density and microglia-specific markers

\begin{tabular}{|c|c|c|c|c|c|c|c|c|c|c|c|c|}
\hline \multirow{2}{*}{ Brain Regions (MSBB) } & \multicolumn{3}{|c|}{ Parahippocampal Gyrus } & \multicolumn{3}{|c|}{ Inferior Frontal Gyrus } & \multicolumn{3}{|c|}{ Frontal Pole } & \multicolumn{3}{|c|}{ Superior Temporal Gyrus } \\
\hline & $\beta$ & SE & $p$-value & $\beta$ & SE & $p$-value & $\beta$ & SE & $p$-value & $\beta$ & SE & $p$-value \\
\hline Plaque Mean Density & 0.0212 & 0.0070 & $3.02 \mathrm{E}-03$ & 0.0163 & 0.0052 & $1.95 \mathrm{E}-03$ & 0.0151 & 0.0059 & $1.22 \mathrm{E}-02$ & 0.0220 & 0.0062 & $5.06 \mathrm{E}-04$ \\
\hline AlF1 & 0.4386 & 0.0811 & 4.10E-07 & 0.2862 & 0.0499 & $6.36 \mathrm{E}-08$ & 0.2179 & 0.0607 & $4.53 \mathrm{E}-04$ & 0.3013 & 0.0572 & $5.36 \mathrm{E}-07$ \\
\hline TMEM119 & 0.7647 & 0.0527 & $<2 \mathrm{E}-16$ & 0.6109 & 0.0446 & $<2 \mathrm{E}-16$ & 0.5062 & 0.0578 & $4.00 \mathrm{E}-15$ & 0.6914 & 0.0496 & $<2 E-16$ \\
\hline
\end{tabular}

Table 2 shows the $\beta$ coefficient $(\beta)$, standard error (SE), and $p$-value for the association analysis between INPP5D expression levels and amyloid plaque density or expression levels of microglia-specific markers AIF1 and TMEM119 by general linear models. 
a

Inpp5d expression (Cortex)

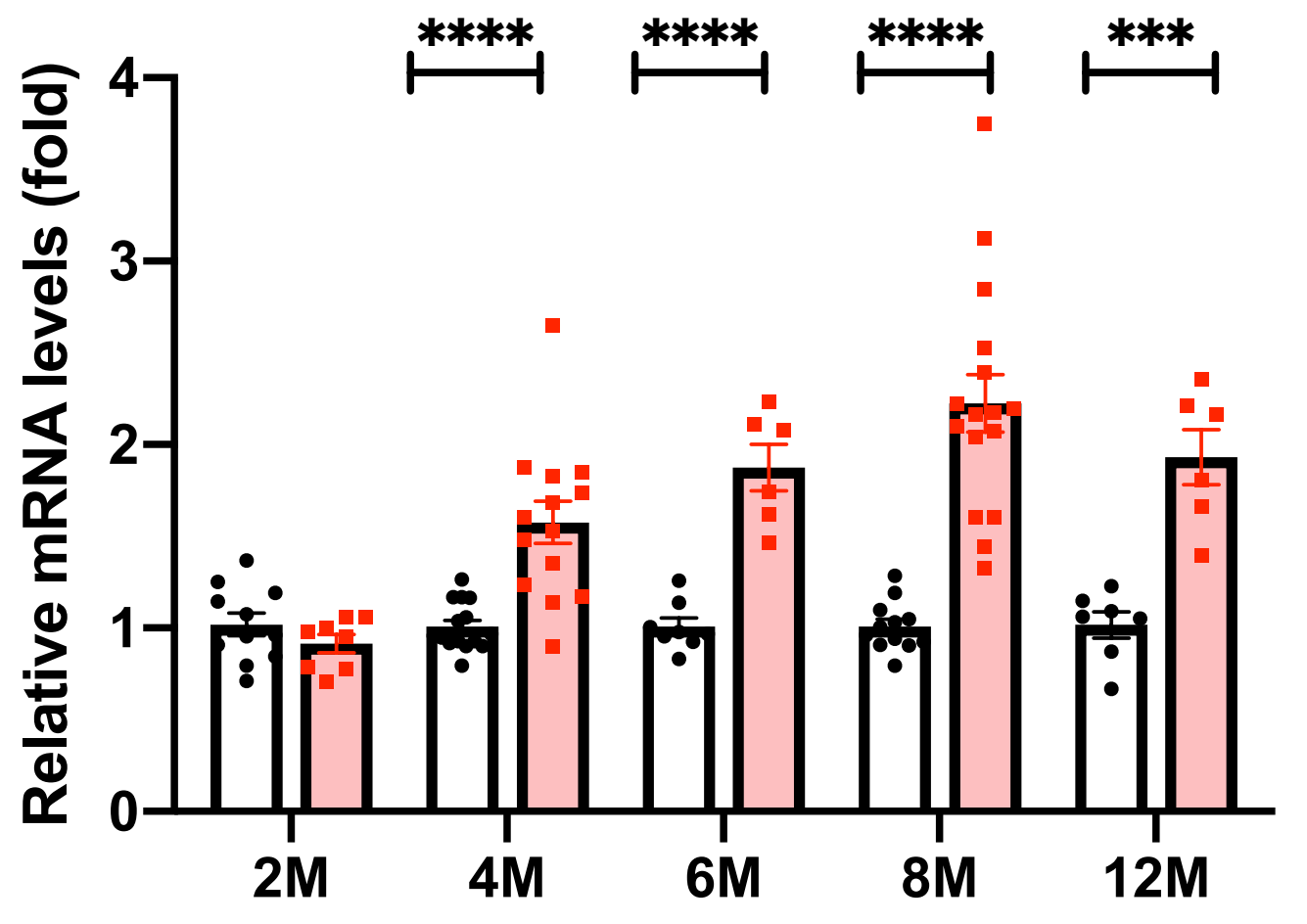

b

Inpp5d expression (Hippocampus)

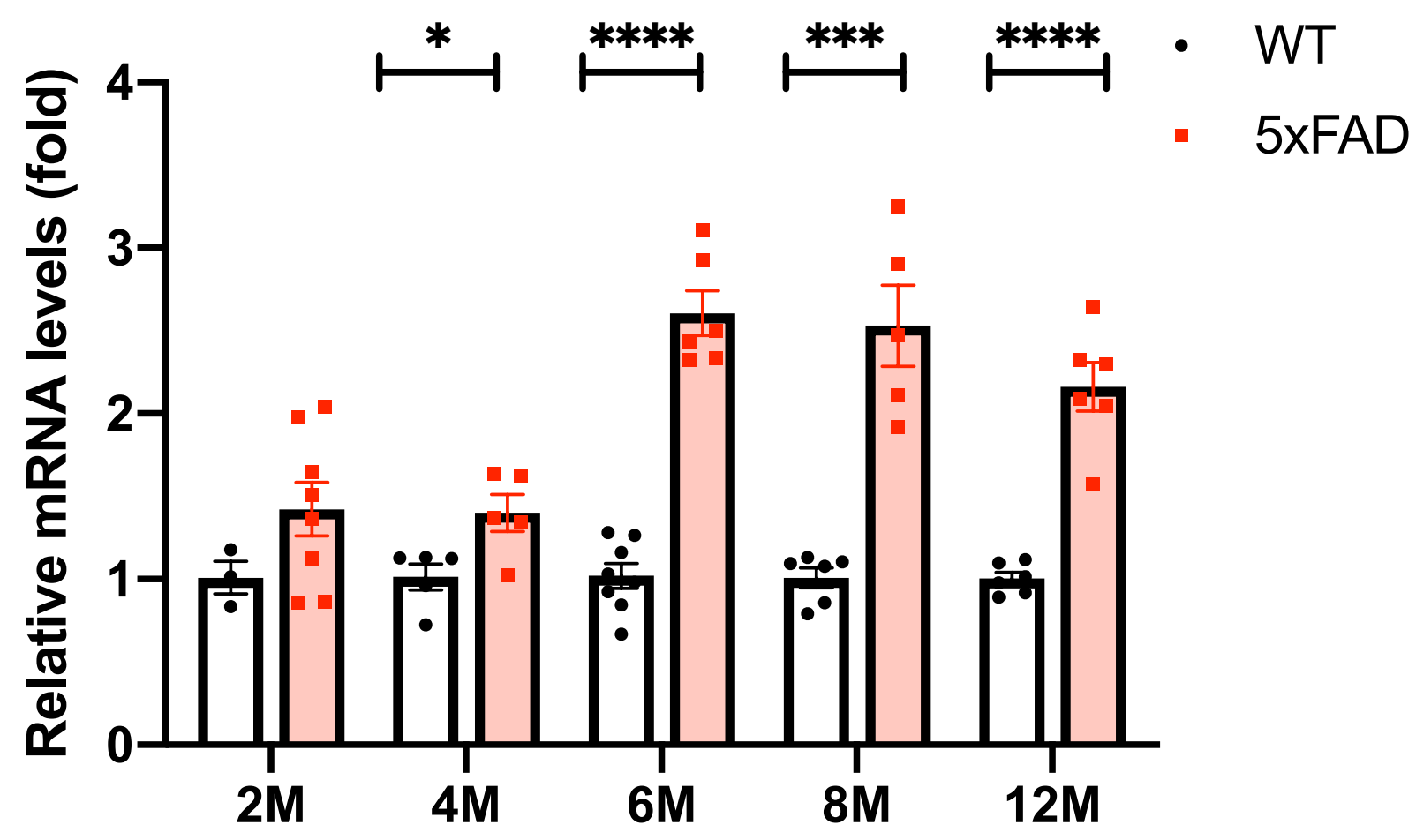


Fig. 3

C

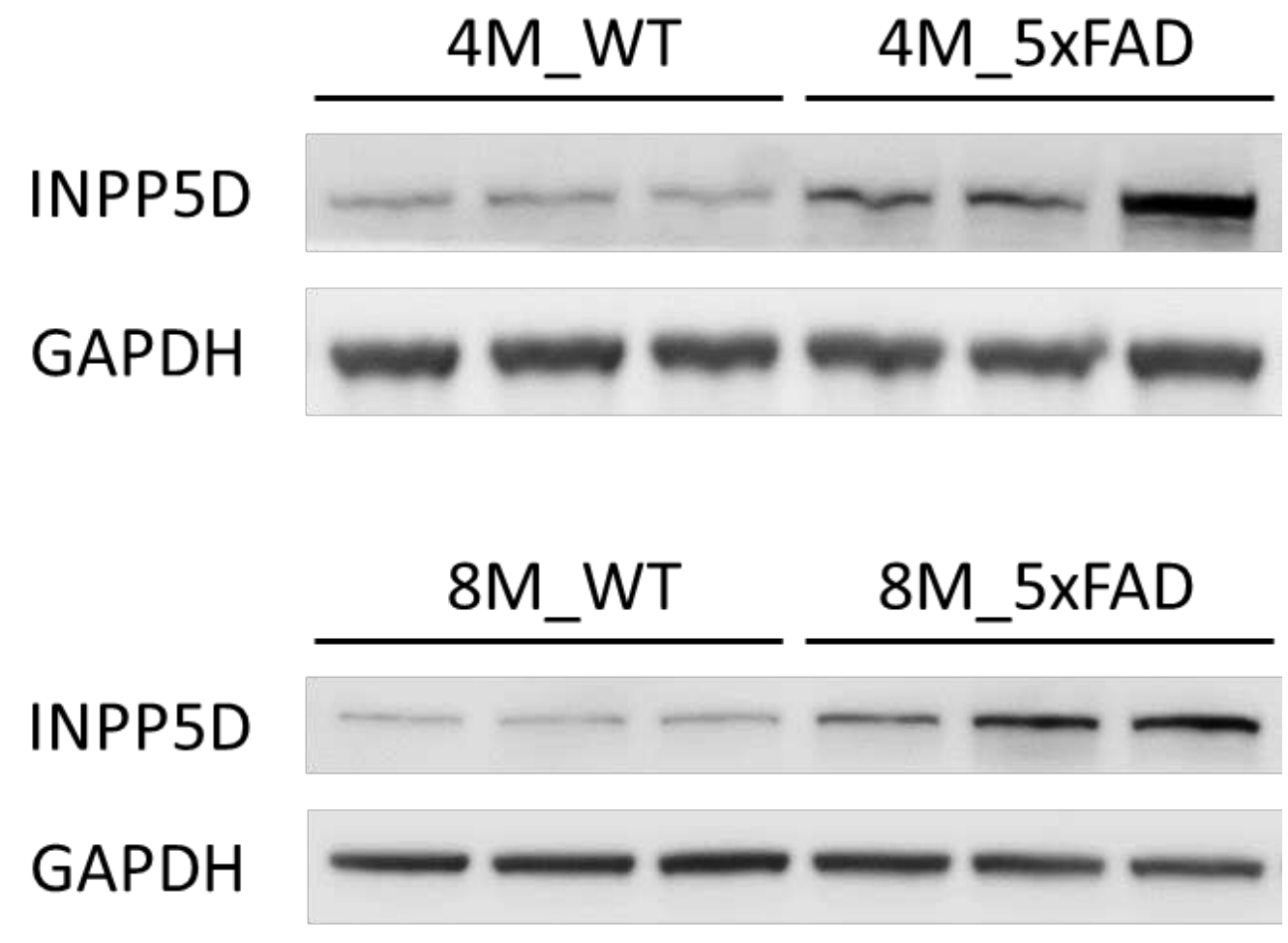

d

INPP5D expression (Cortex)

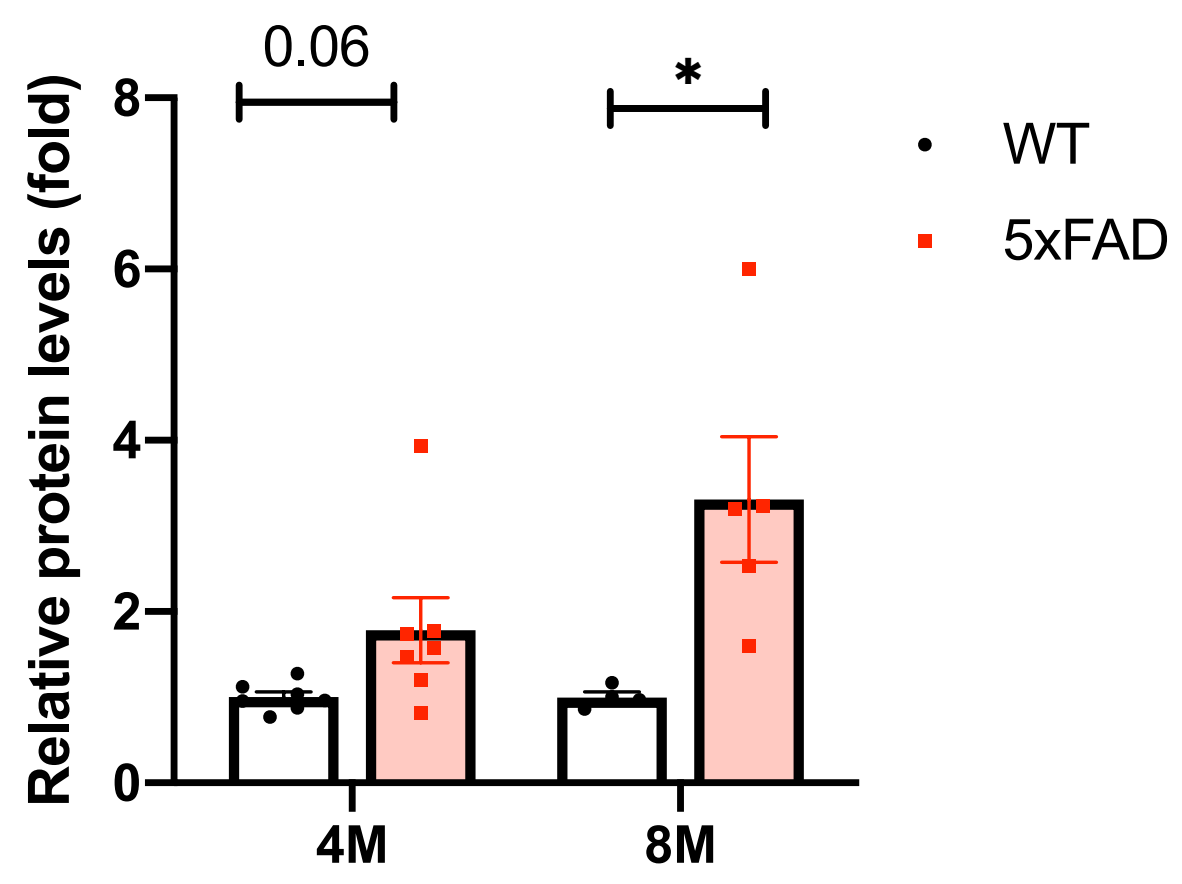


e
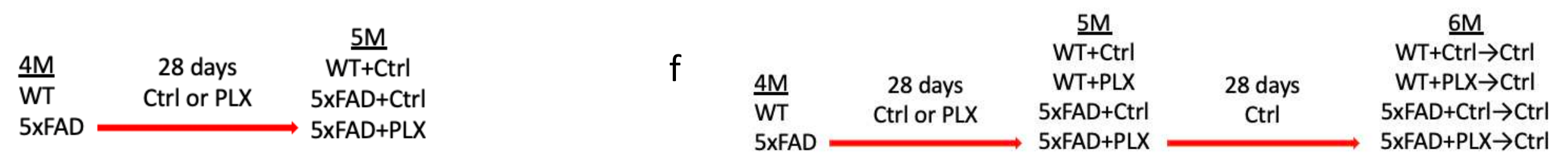

Inpp5d expression (Cortex)

Inpp5d expression (Cortex)
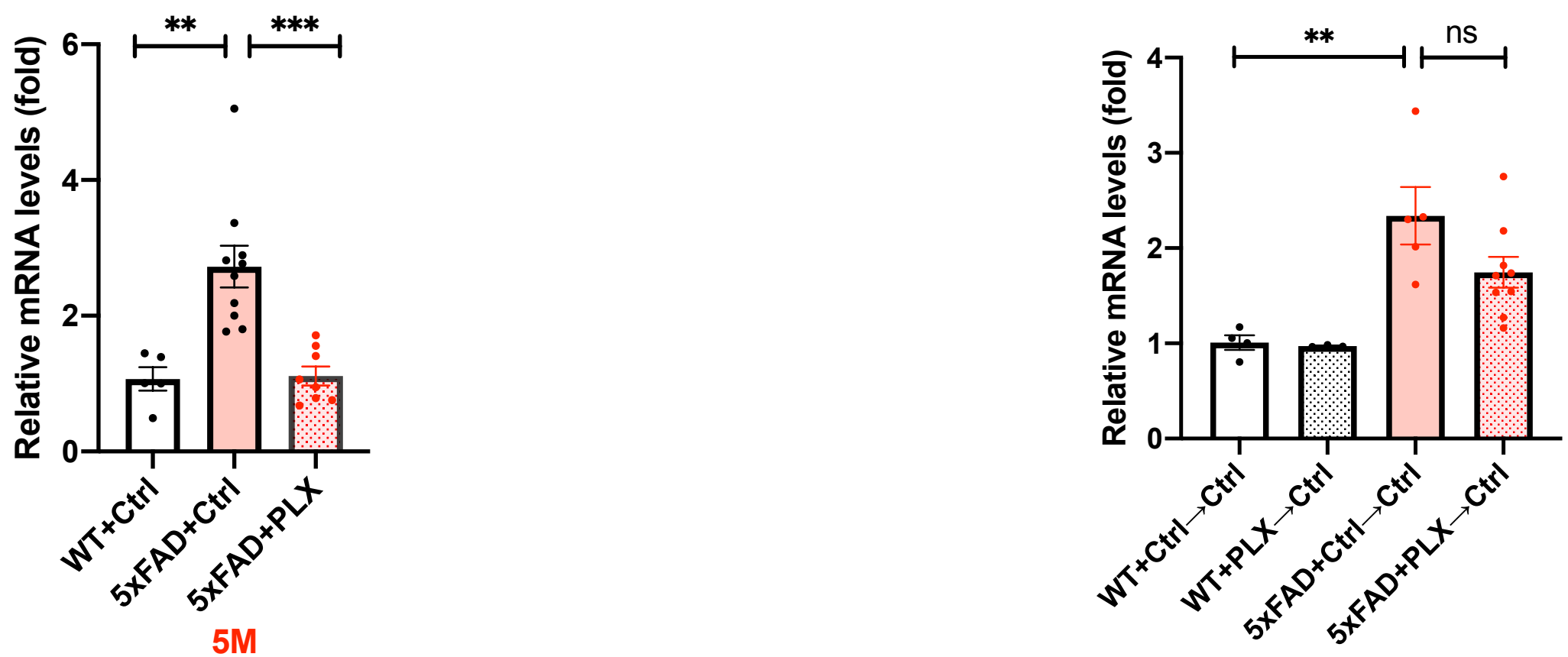

$6 \mathrm{M}$

Fig 3. Inpp5d levels are increased in 5xFAD mice

Gene and protein levels of Inpp5d were assessed in cortical and hippocampal lysates from 5xFAD mice. Gene expression levels of Inpp5d were significantly increased in both cortex (a) and hippocampus (b) at 4, 6, 8, and 12 months of age ( $\mathrm{n}=6$-15 mice). There were significant changes in Inpp5d protein levels in the cortex at 8 months of age and an increased trend in the cortex at 4 months of age ( $n=4-7)$. Increased Inpp5d levels were abolished with PLX5622 treatment (e), and restored after switching PLX diet to normal diet $(f)(n=3-10)$. ${ }^{*} p<0.05$; ${ }^{* *} p<0.01$; ${ }^{* * *} p<0.001$; $* * * * p<0.0001$, ns not significant. 
Fig. 4 INPP5D expression levels are increased in plaque-associated microglia.

a Cortex

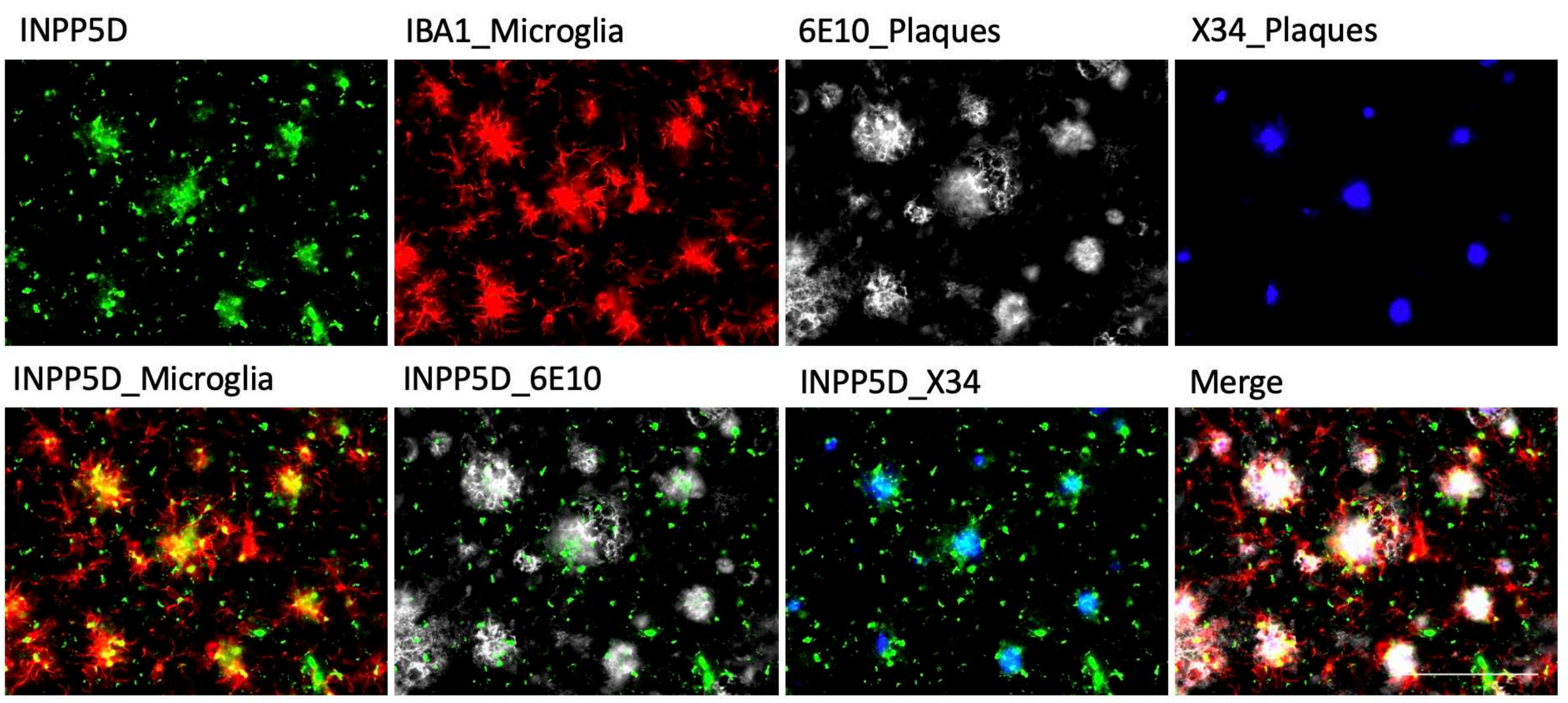


b Subiculum

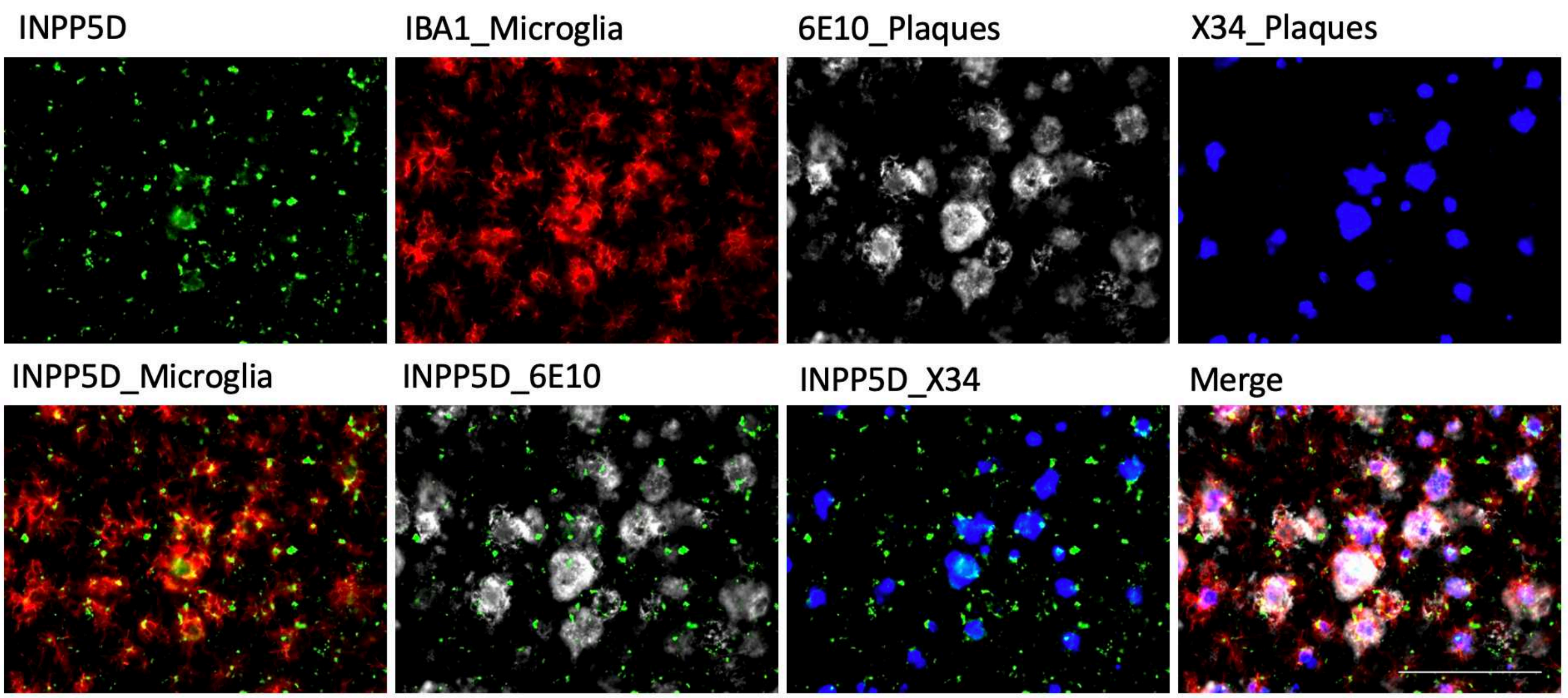




\section{Sorted microglia RNA-Seq}

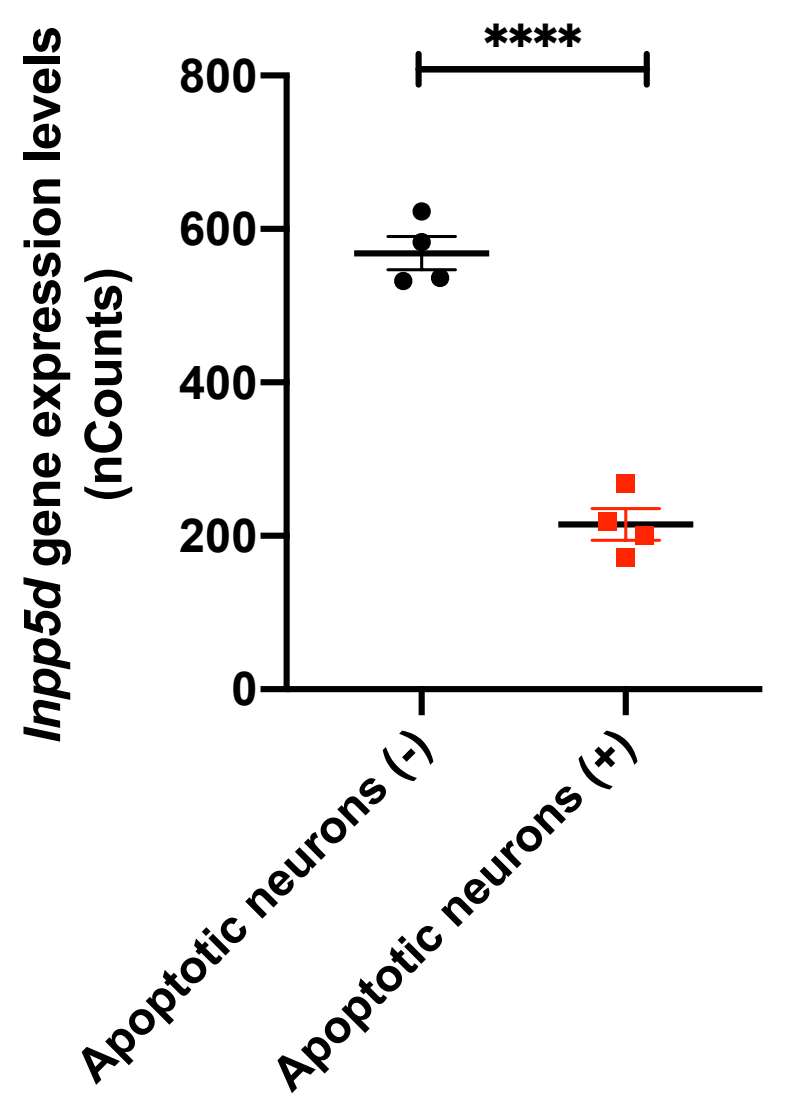

Fig 4. INPP5D expression levels were increased in plaque-associated microglia. INPP5D was mainly expressed in plaque-associated microglia. INPP5D- and IBA1 (AIF1)-positive microglia cluster around 6E10-positive or X-34-positive plaques in both cortex (a) and subiculum (b) of 8-month-old mice. Analysis of transcriptomic data of sorted microglia from wild-type mice cortex-injected labeled apoptotic neurons revealed that Inpp5d expression is increased in non-phagocytic microglia (Krasemann et.al) (c). Scale bar, 10 $\mu \mathrm{m} .{ }^{* * *} \mathrm{p}<0.0001$ 
Figures

b

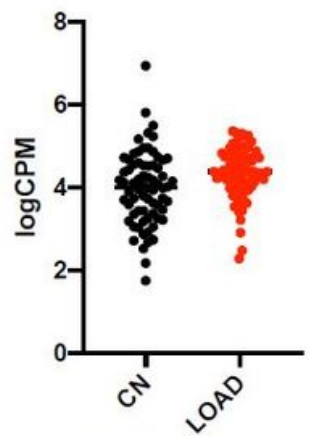

Temporal cortex

e

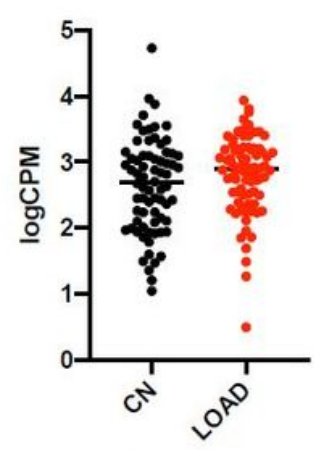

Cerebellum
C

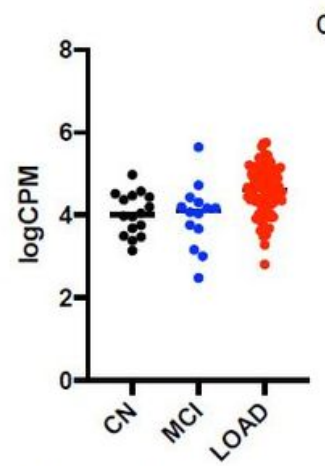

Parahippocampal gyrus

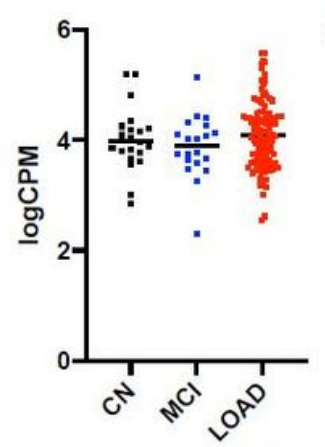

Frontal pole

d

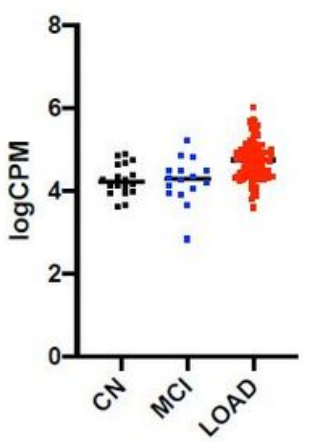

Inferior frontal gyrus

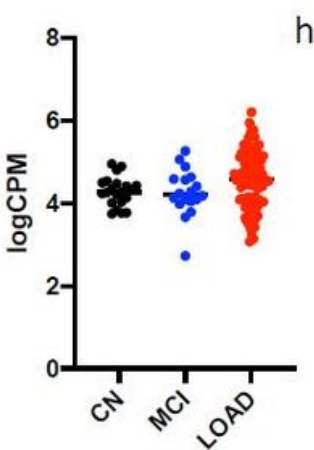

Superior temporal gyrus

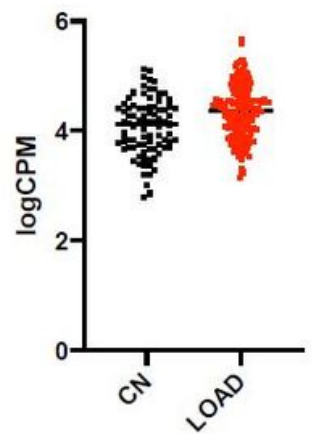

Dorsolateral prefrontal cortex

$\mathrm{CN}$ cognitively normal, $\mathrm{MCl}$ mild cognitive impairment, LOAD Late-Onset Alzheimer's disease

Figure 1

Relative quantification of INPP5D expression in the studied participants 
a
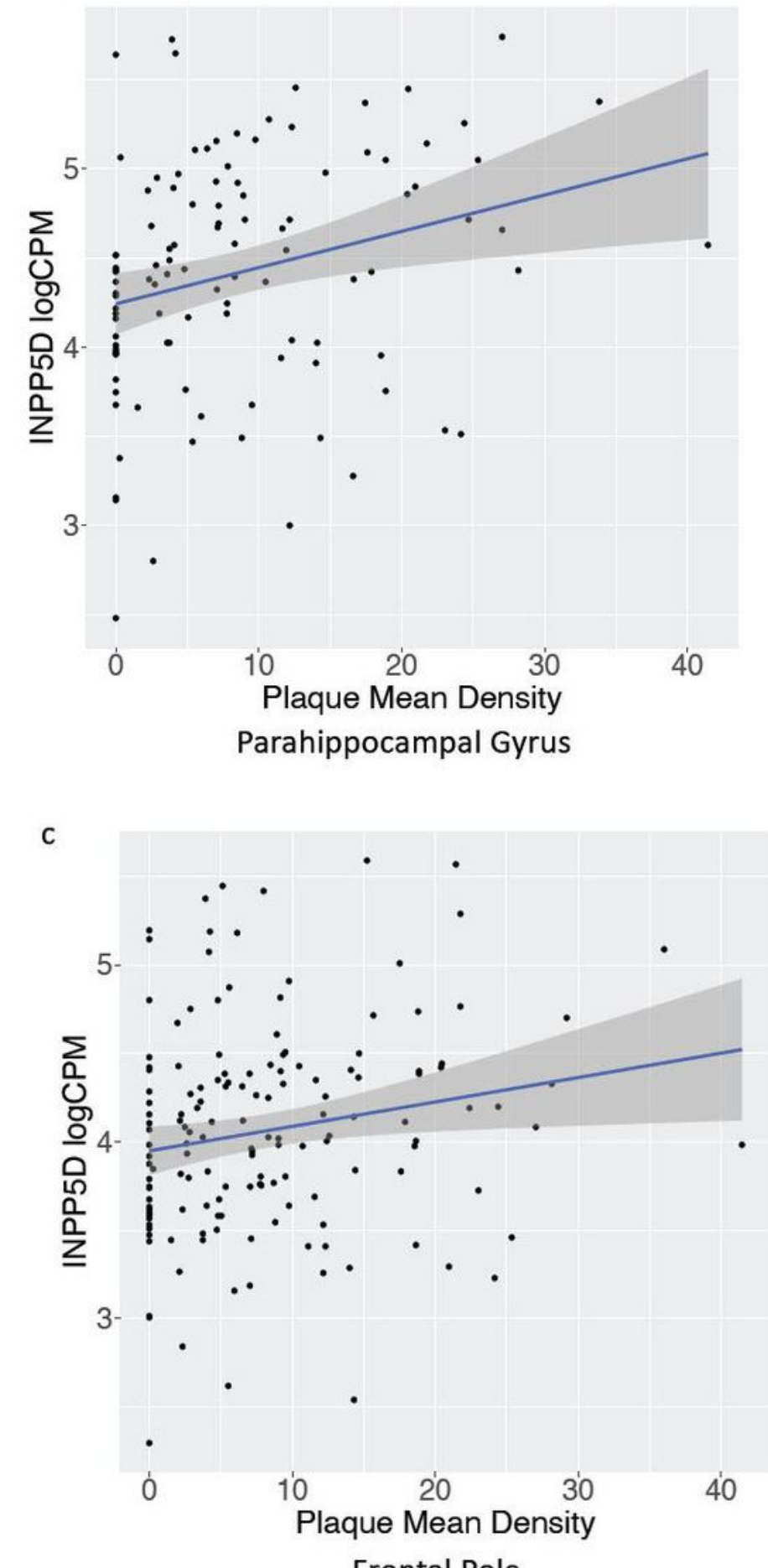

Frontal Pole b
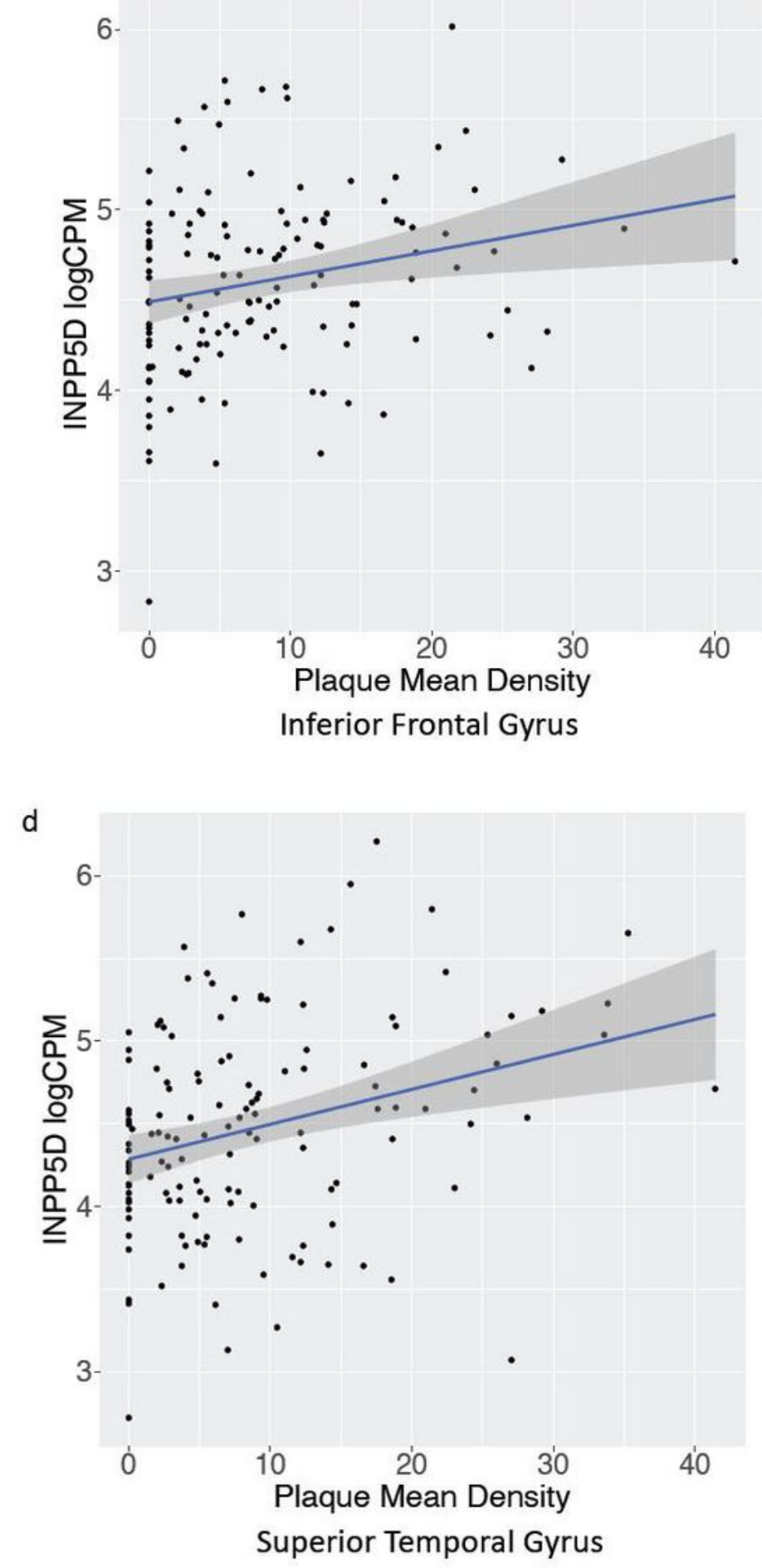

\section{Figure 2}

Association of INPP5D expression with amyloid plaque mean density The scatter plots show the positive association between INPP5D expression and plaque mean density in (a) parahippocampal gyrus, (b) inferior frontal gyrus, (c) frontal pole, and (d) superior temporal gyrus from the MSBB cohort. 
a

Inpp5d expression (Cortex)

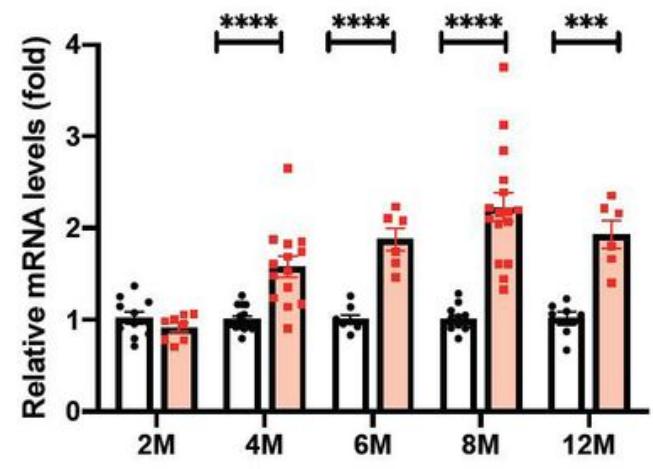

C

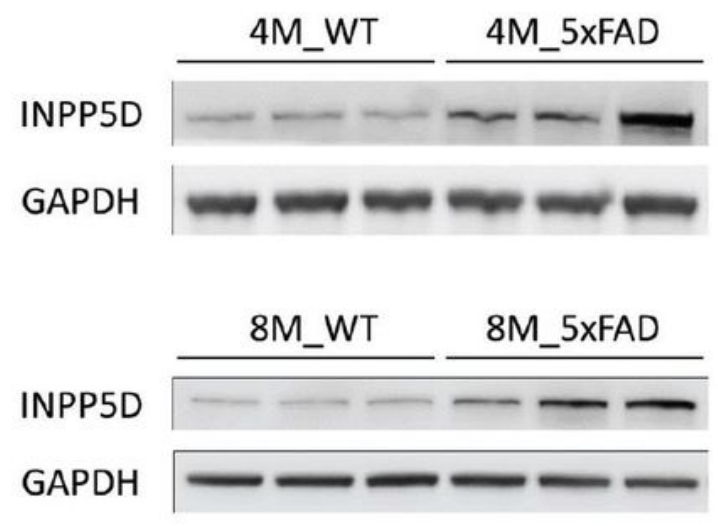

e

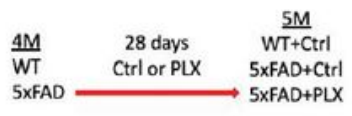

Inpp5d expression (Cortex)

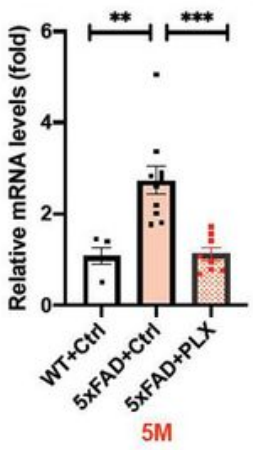

Inpp5d expression (Hippocampus)

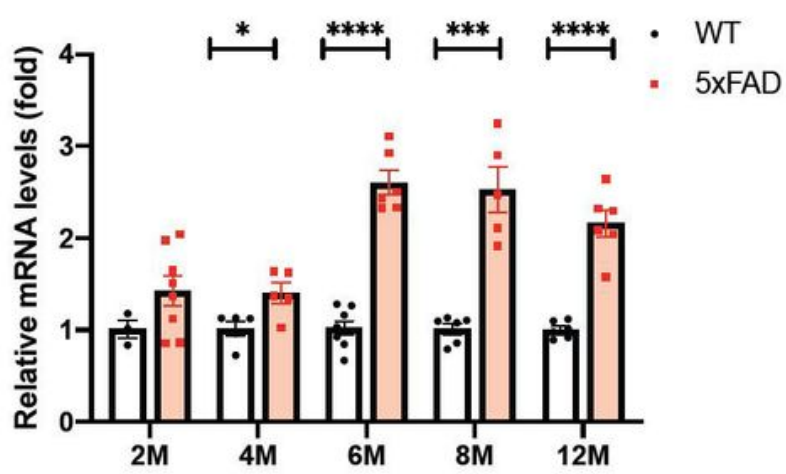

d
INPP5D expression (Cortex)
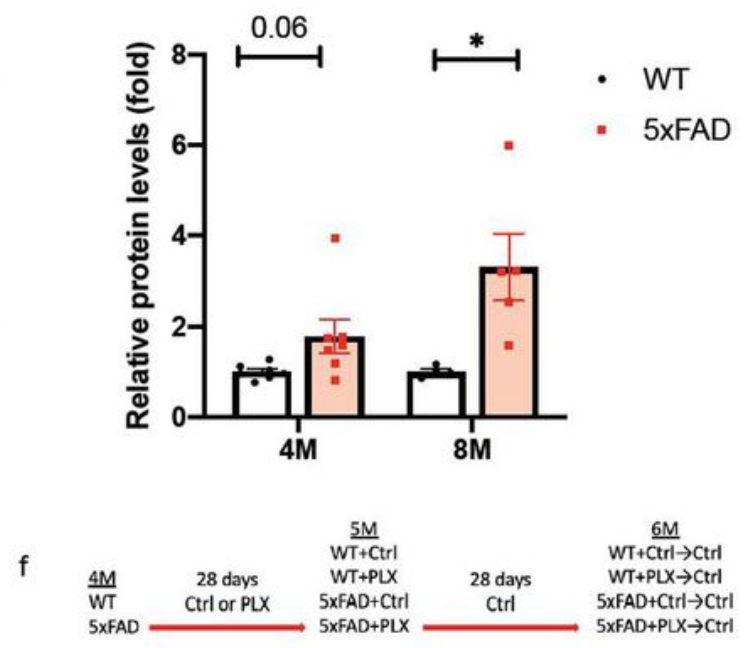

Inpp5d expression (Cortex)

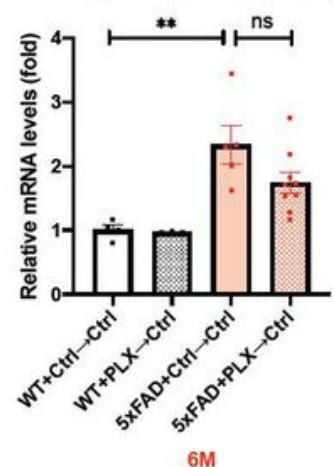

Figure 3

Inpp5d levels are increased in 5xFAD mice Gene and protein levels of Inpp5d were assessed in cortical and hippocampal lysates from 5xFAD mice. Gene expression levels of Inpp5d were significantly increased in both cortex (a) and hippocampus (b) at 4, 6, 8, and 12 months of age ( $n=6-15$ mice). There were significant changes in Inpp5d protein levels in the cortex at 8 months of age and an increased trend in the cortex at 4 months of age $(n=4-7)$. Increased Inpp5d levels were abolished with PLX5622 treatment 
(e), and restored after switching PLX diet to normal diet (f) $(n=3-10) .{ }^{*} p<0.05 ;{ }^{* \star} p<0.01 ;{ }^{* \star *} p<0.001$; $\star \star \star * p<0.0001$, ns not significant.

\section{a Cortex}

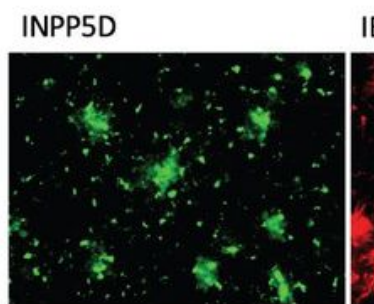

IBA1_Microglia

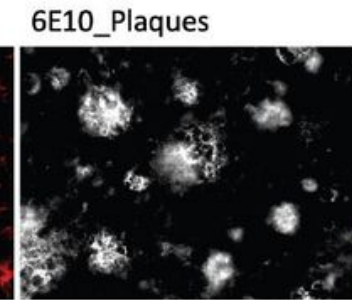

X34_Plaques

INPP5D_Microglia
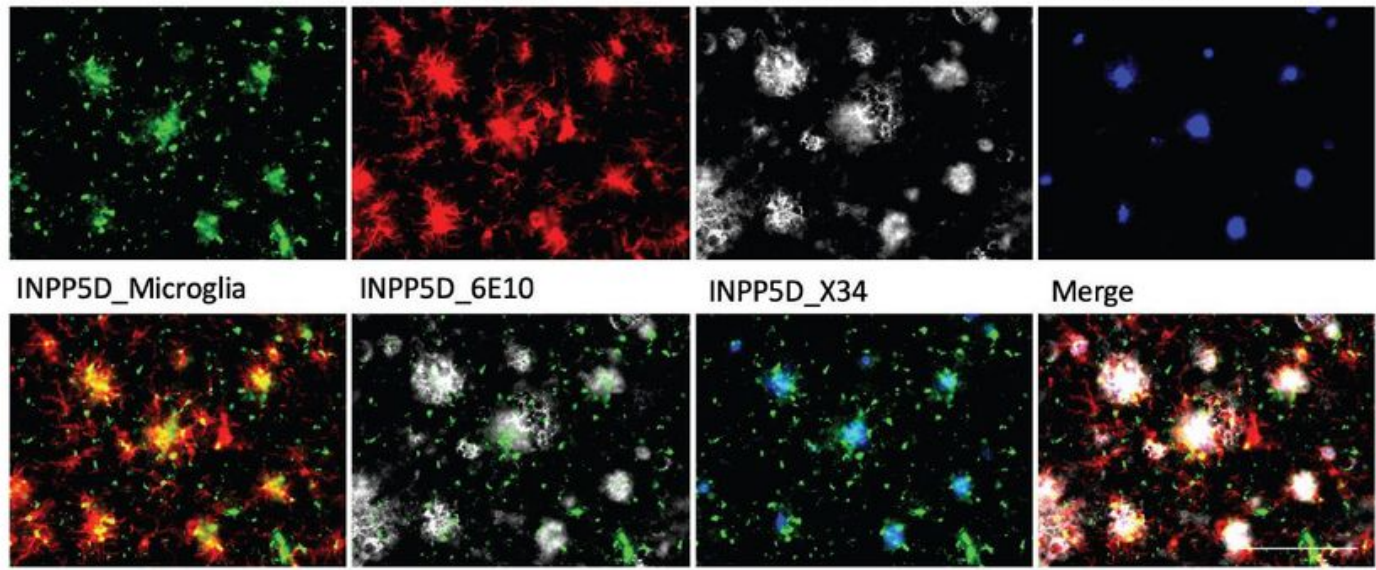

INPP5D_6E10

INPP5D_X34

Merge
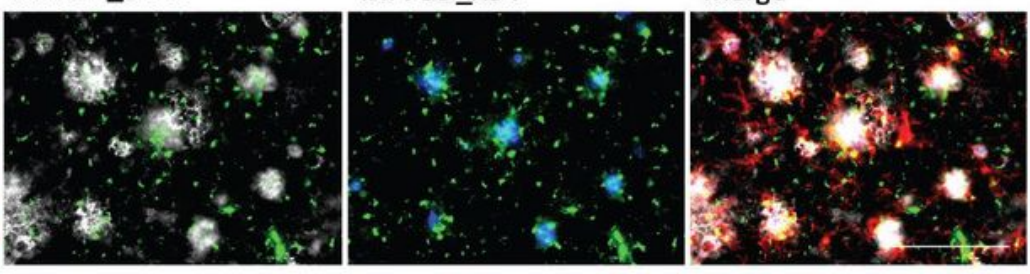

b Subiculum

INPP5D

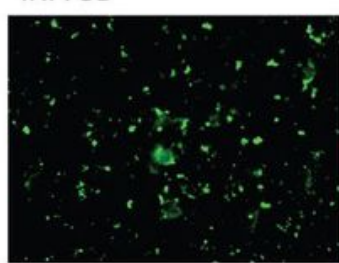

IBA1_Microglia
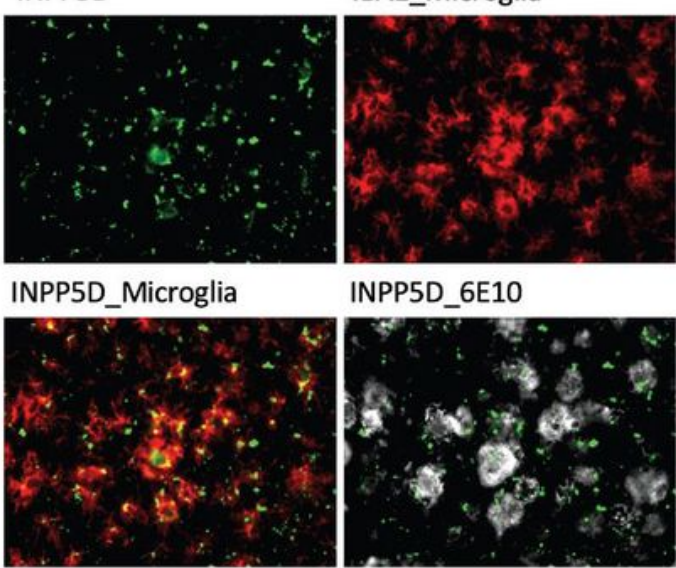

INPP5D_6E10

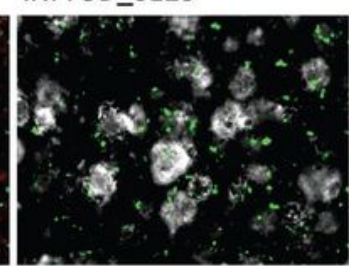

6E10 Plaques

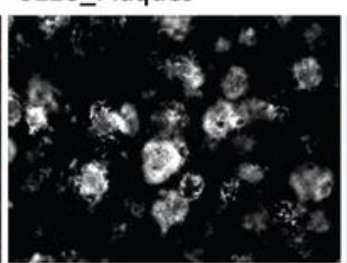

INPP5D_X34

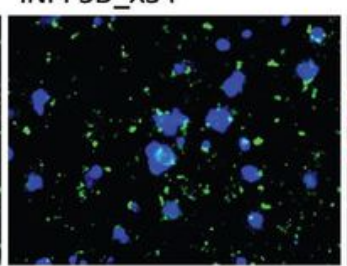

X34_Plaques

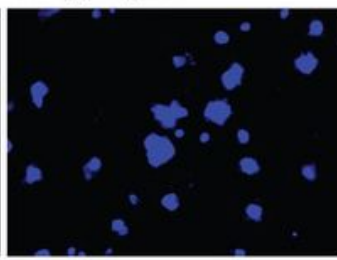

Merge

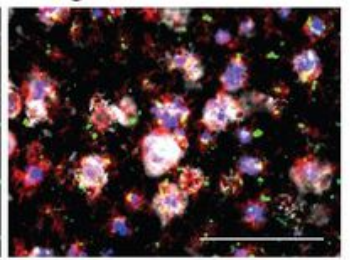

c

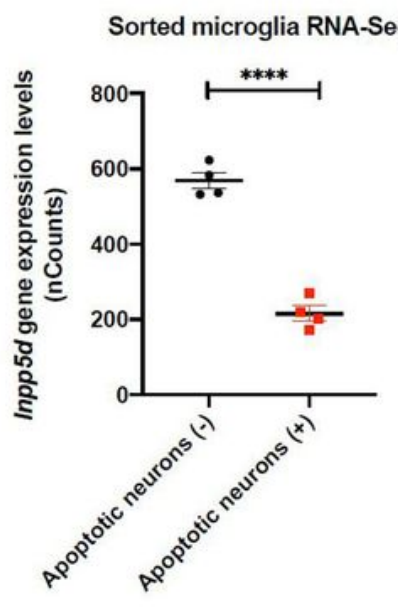

Fig 4. INPP5D expression levels were increased in plaque-associated microglia. INPP5D was mainly expressed in plaque-associated microglia. INPP5D- and IBA1 (AIF1)-positive microglia cluster around 6E10-positive or X-34-positive plaques in both cortex (a) and subiculum (b) of 8-month-old mice. Analysis of transcriptomic data of sorted microglia from wild-type mice cortex-injected labeled apoptotic neurons revealed that Inpp5d expression is increased in non-phagocytic microglia (Krasemann et.al) (c). Scale bar, 10 $\mu \mathrm{m} .{ }^{* * * *} \mathrm{p}<0.0001$

\section{Figure 4}

INPP5D expression levels are increased in plaque-associated microglia. INPP5D was mainly expressed in plaque-associated microglia. INPP5D- and IBA1 (AIF1)-positive microglia cluster around 6E10-positive or X-34-positive plaques in both cortex (a) and subiculum (b) of 8-month-old mice. Analysis of 
transcriptomic data of sorted microglia from wild-type mice cortex-injected labeled apoptotic neurons revealed that Inpp5d expression is increased in non-phagocytic microglia (Krasemann et.al) (c). Scale bar, $10 \mu \mathrm{m} . * \star \star * \mathrm{p}<0.0001$ 